\title{
The optimal vaccination strategy to control COVID-19: a modeling study in Wuhan City, China
}

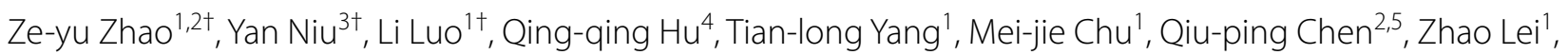
Jia Rui ', Cheng-long Song ${ }^{6}$, Sheng-nan Lin' ${ }^{1}$, Yao Wang ${ }^{1}$, Jing-wen $X u^{1}$, Yuan-zhao Zhu' ${ }^{1}$ Xing-chun Liu', Meng Yang ${ }^{1}$, Jie-feng Huang ${ }^{1}$, Wei-kang Liu' ${ }^{1}$, Bin Deng ${ }^{1}$, Chan Liu' ${ }^{1}$, Zhuo-yang Li ', Pei-hua Li', Yan-hua Su', Ben-hua Zhao', Wen-long Huang ${ }^{7^{*}}$, Roger Frutos ${ }^{2^{*}}$ and Tian-mu Chen ${ }^{1 *}$ (1)

\begin{abstract}
Background: Reaching optimal vaccination rates is an essential public health strategy to control the coronavirus disease 2019 (COVID-19) pandemic. This study aimed to simulate the optimal vaccination strategy to control the disease by developing an age-specific model based on the current transmission patterns of COVID-19 in Wuhan City, China.

Methods: We collected two indicators of COVID-19, including illness onset data and age of confirmed case in Wuhan City, from December 2, 2019, to March 16, 2020. The reported cases were divided into four age groups: group 1, $\leq 14$ years old; group 2, 15 to 44 years old; group 3, 44 to 64 years old; and group 4, $\geq 65$ years old. An age-specific susceptible-exposed-symptomatic-asymptomatic-recovered/removed model was developed to estimate the transmissibility and simulate the optimal vaccination strategy. The effective reproduction number $\left(R_{\text {eff }}\right)$ was used to estimate the transmission interaction in different age groups.

Results: A total of 47722 new cases were reported in Wuhan City from December 2, 2019, to March 16, 2020. Before the travel ban of Wuhan City, the highest transmissibility was observed among age group $2\left(R_{\text {eff }}=4.28\right)$, followed by group 2 to $3\left(R_{\text {eff }}=2.61\right)$, and group 2 to $4\left(R_{\text {eff }}=1.69\right)$. China should vaccinate at least $85 \%$ of the total population to interrupt transmission. The priority for controlling transmission should be to vaccinate $5 \%$ to $8 \%$ of individuals in age group 2 per day (ultimately vaccinated $90 \%$ of age group 2), followed by 10\% of age group 3 per day (ultimately vaccinated $90 \%$ age group 3). However, the optimal vaccination strategy for reducing the disease severity identified individuals $\geq 65$ years old as a priority group, followed by those $45-64$ years old.
\end{abstract}

\footnotetext{
*Correspondence: 497528061@qq.com; frutossmt@gmail.com; 13698665@qq.com

†Ze-yu Zhao, Yan Niu and Li Luo contributed equally to this study

1 State Key Laboratory of Molecular Vaccinology and Molecular

Diagnostics, School of Public Health, Xiamen University, 4221-117 South

Xiang'an Road, Xiang'an District, Xiamen 361102, Fujian, People's Republic

of China

${ }^{2}$ Cirad, UMR 17, Intertryp, Université de Montpellier, Montpellier, France

${ }^{7}$ Fujian Provincial Center for Disease Control and Prevention, 76 Jintai

Road, Gulou District, Fuzhou, Fujian, People's Republic of China

Full list of author information is available at the end of the article
}

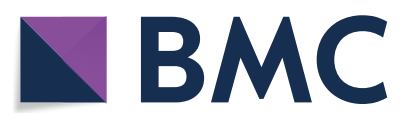

(c) The Author(s) 2021. Open Access This article is licensed under a Creative Commons Attribution 4.0 International License, which permits use, sharing, adaptation, distribution and reproduction in any medium or format, as long as you give appropriate credit to the original author(s) and the source, provide a link to the Creative Commons licence, and indicate if changes were made. The images or other third party material in this article are included in the article's Creative Commons licence, unless indicated otherwise in a credit line to the material. If material is not included in the article's Creative Commons licence and your intended use is not permitted by statutory regulation or exceeds the permitted use, you will need to obtain permission directly from the copyright holder. To view a copy of this licence, visit http://creativecommons.org/licenses/by/4.0/. The Creative Commons Public Domain Dedication waiver (http://creativeco mmons.org/publicdomain/zero/1.0/) applies to the data made available in this article, unless otherwise stated in a credit line to the data. 
Conclusions: Approximately $85 \%$ of the total population (nearly 1.2 billion people) should be vaccinated to build an immune barrier in China to safely consider removing border restrictions. Based on these results, we concluded that 90\% of adults aged 15-64 years should first be vaccinated to prevent transmission in China.

Keywords: SARS-CoV-2, Transmissibility, Age-specific model, Vaccination strategy, Effectiveness

\section{Graphical Abstract}

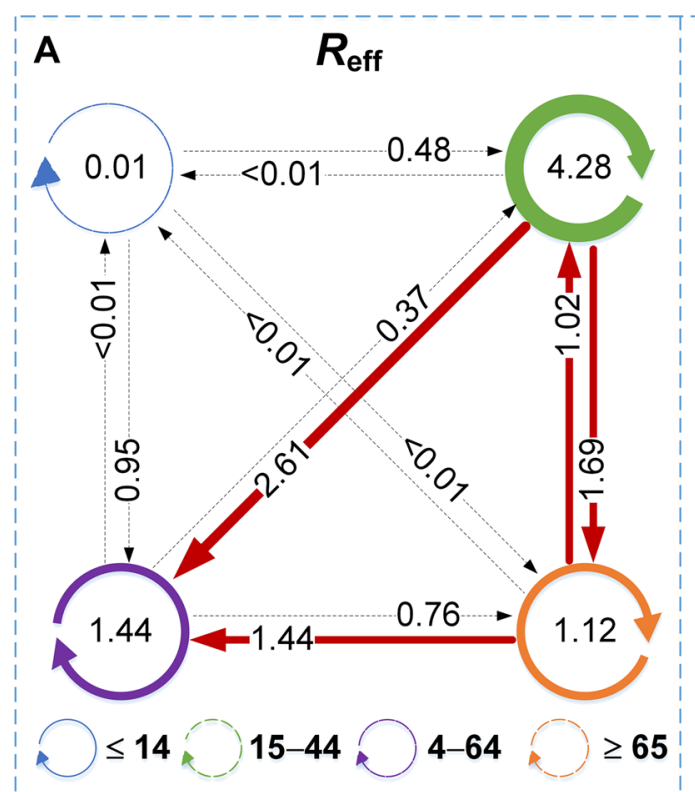

\section{C}

$$
\leq 14
$$

$$
\text { 15-44 }
$$

을 45-64

क D

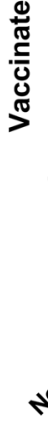

$\leq 14$

$15-44$

$45-64$

$\geq 65$
Total population
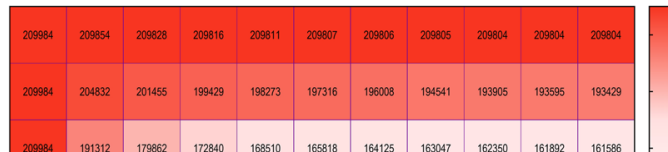

\begin{tabular}{|l|l|l|l|l|l|l|l|l|l|}
\hline 191312 & 179862 & 172840 & 168550 & 165813 & 164125 & 163047 & 162350 & 161892 & 166593 \\
\hline
\end{tabular}

\begin{tabular}{|l|l|l|l|l|l|l|l|l|l|}
\hline 181718 & 163848 & 152555 & 145392 & 140016 & 137864 & 135939 & 134666 & 133812 & 133230 \\
\hline
\end{tabular}

$\geq 65$ years old

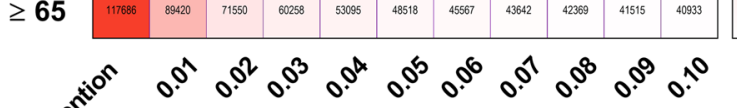

Vaccination rate

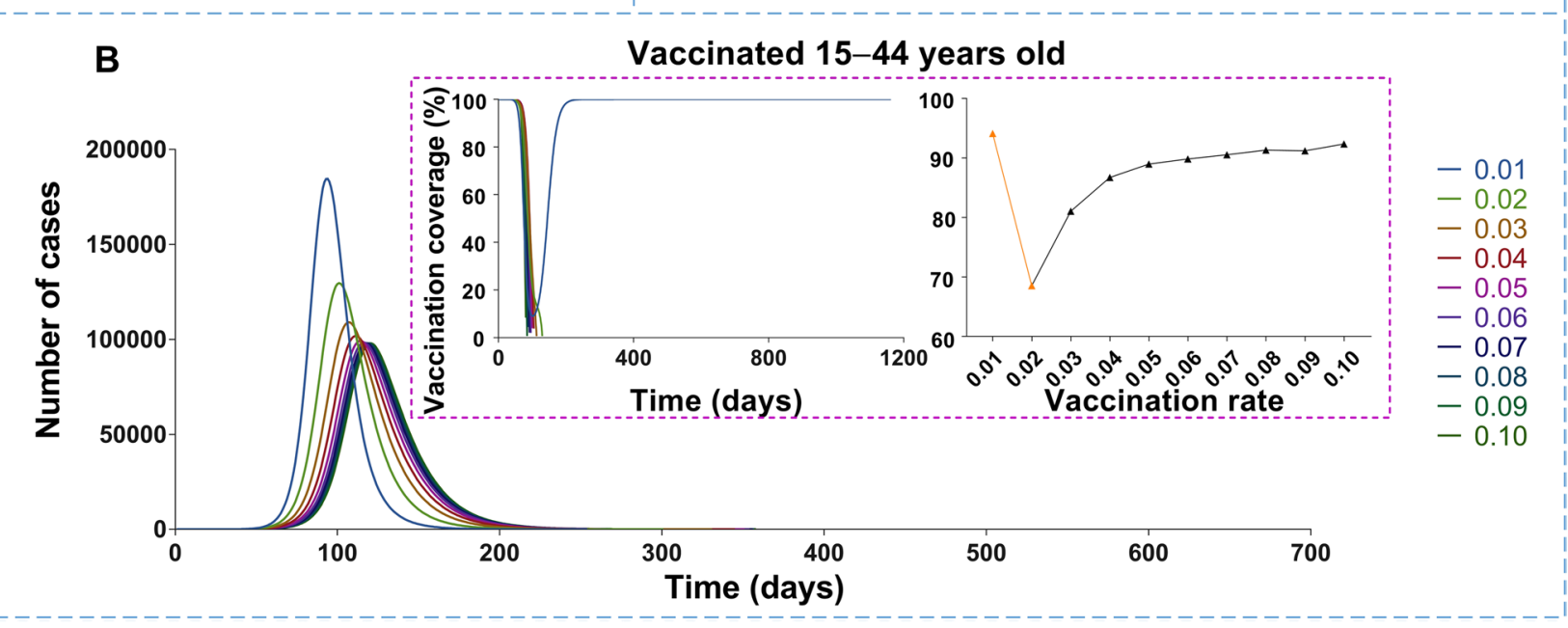

\section{Background}

Currently, the coronavirus disease 2019 (COVID-19) pandemic has become a heavy burden worldwide. As of November 15, 2021, 253163330 confirmed cases have been reported by the World Health Organization [1]. Several effective non-pharmaceutical interventions (NPIs) such as travel bans, social distancing, case isolation, and mask-wearing have been applied in China to mitigate the epidemic [2]. However, the implementation of NPIs has varied considerably across countries
[3]. Therefore, vaccine and antiviral therapies are essential to prevent the spread of COVID-19 and control the epidemic [4]. Recently, studies have shown that the vaccine efficacy (VE) ranged from 90 to $94 \%$ (with two doses of vaccination) for controlling infection of the ancestral virus [5-7], and even $67 \%$ to $88 \%$ (with two doses) for the Delta variant [8]. Furthermore, the vaccine's effectiveness for controlling disease severity and death was higher than that for reducing infection, especially with the great heterogeneity in different age 
groups [9]. Therefore, optimal vaccination protocols for different age groups should be simulated to help guide an appropriate vaccine strategy.

Most current studies used dynamic models of severe acute respiratory syndrome coronavirus 2 (SARS-CoV-2), such as the susceptible-exposed-infectious-removed (SEIR) model to clarify early transmission, and the multi-host model to estimate transmissibility [10, 11]. Moreover, our previous study adopted an age-specific susceptible-exposed-symptomatic-asymptomatic-recovered/removed (SEIAR) model to estimate the relative transmissibility in different age groups, but did not simulate the vaccine effects [12]. Furthermore, the other study adopted a SEIR model to access the vaccine effects in different populations suggested that a $\mathrm{VE} \geq 50 \%$ would be sufficient to mitigate the pandemic [13]. Some modeling studies recommended targeting older age groups as an optimal strategy for controlling death [14, 15], which was suitable for reducing the disease severity and death rates in some Western countries. Furthermore, some studies have simulated vaccine effectiveness based on the contact matrix and assumption of basic reproduction number $\left(R_{0}\right)[13,15,16]$, but did not analyze the transmission pattern in different age groups through first-hand data. In this study, we employed an age-specific SEIAR model to explore the transmission features, compare the transmissibility and assess the vaccine effectiveness in different age groups.

\section{Methods \\ Data collection and study design}

Data including age and date of onset of COVID-19 in Wuhan City from December 2, 2019, to March 16, 2020, were collected from a previous study [17] (Additional file 1: Table S1). Our study included four phases including model development, parameter estimation, transmission assessment, and vaccination simulation (Fig. 1). The subscripts $i$ and $j(i \neq j)$ are referred to as age groups 1 to 4 , respectively. Age group 1 was defined as $\leq 14$ years old; group 2, 15-44 years old; group 3, 45-64 years old; and group $4, \geq 65$ years old.

\section{Model development}

We adopted an age-specific SEIAR model to estimate the transmissibility (Model 1), and two vaccinated age-specific SEIAR models (Model 2 and Model 3) to simulate the vaccination effects.

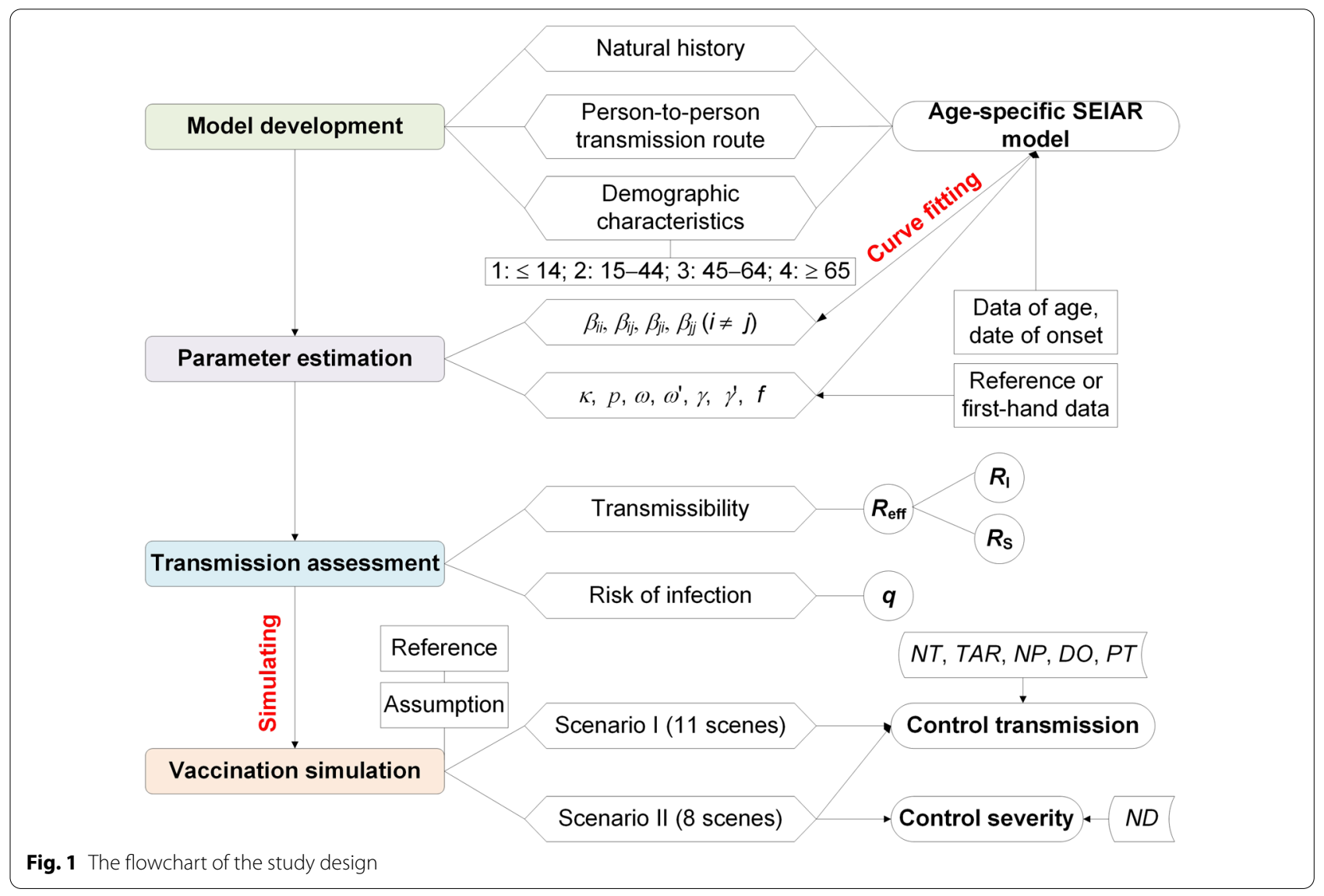


According to our previous study, we developed a multihost model and age-specific model and further applied the age-specific SEIAR model to estimate the disease transmission $[11,12]$. In the model, the total population $(N)$ was divided into five categories, susceptible $(S)$, exposed $(E)$, symptomatic $(I)$, asymptomatic $(A)$, and recovered/ removed $(R)$. Table 1 presents the definitions in detail.

The model conditions or assumptions were as follows:

(a) Susceptible individuals infected by contact with symptomatic or asymptomatic patients. SARS$\mathrm{CoV}-2$ can be transmitted within a given age group $i$ with a relative transmission rate $\left(\beta_{i i}\right)$ and between

Table 1 Variables in the age-specific model

\begin{tabular}{lll}
\hline Variables & Description & Unit \\
\hline $\mathrm{S}$ & Susceptible individuals & Individuals \\
$\mathrm{E}$ & Exposed individuals & Individuals \\
$\mathrm{I}$ & Infectious individuals & Individuals \\
$\mathrm{A}$ & Asymptomatic individuals & Individuals \\
$\mathrm{R}$ & Recovered/Removed individuals & Individuals \\
$\mathrm{V}_{1}$ & Vaccinated individuals without immunity & Individuals \\
$\mathrm{V}_{2}$ & Vaccinated individuals with immunity & Individuals \\
$\mathrm{N}$ & Total number of population & Individuals \\
\hline
\end{tabular}

age groups $i$ and $j$ with a relative transmission rate $\left(\beta_{i j}\right)$.

(b) The proportion of asymptomatic infections was defined as $p$. The exposed individuals would become symptomatic and asymptomatic after an incubation period $(1 / \omega)$ and a latent period $\left(1 / \omega^{\prime}\right)$. In the model, the incubation period was assumed to be equal to the latent period.

(c) The transmissibility of the virus from asymptomatic and symptomatic patients differs by factor $\kappa$.

(d) The symptomatic and asymptomatic patients are converted to recovered/removed persons after an infectious period of $1 / \gamma$ and $1 / \gamma^{\prime}$, respectively.

A flowchart of Model 1 is presented in Fig. 2. The equations of the model are as follows:

$$
i \neq j
$$

$$
\begin{aligned}
\frac{d S_{i}}{d t}= & -\beta_{i i} S_{i}\left(I_{i}+\kappa A_{i}\right)-\beta_{j i} S_{i}\left(I_{j}+\kappa A_{j}\right)-\delta_{i} S_{i} \\
\frac{d E_{i}}{d t}= & \beta_{i i}\left(V_{1 i}+(1-\lambda) V_{2 i}+S_{i}\right)\left(I_{i}+\kappa A_{i}\right) \\
& +\beta_{j i}\left(V_{1 i}+(1-\lambda) V_{2 i}+S_{i}\right)\left(I_{j}+\kappa A_{j}\right) \\
& -(1-p) \omega E_{i}-p \omega^{\prime} E_{i}
\end{aligned}
$$

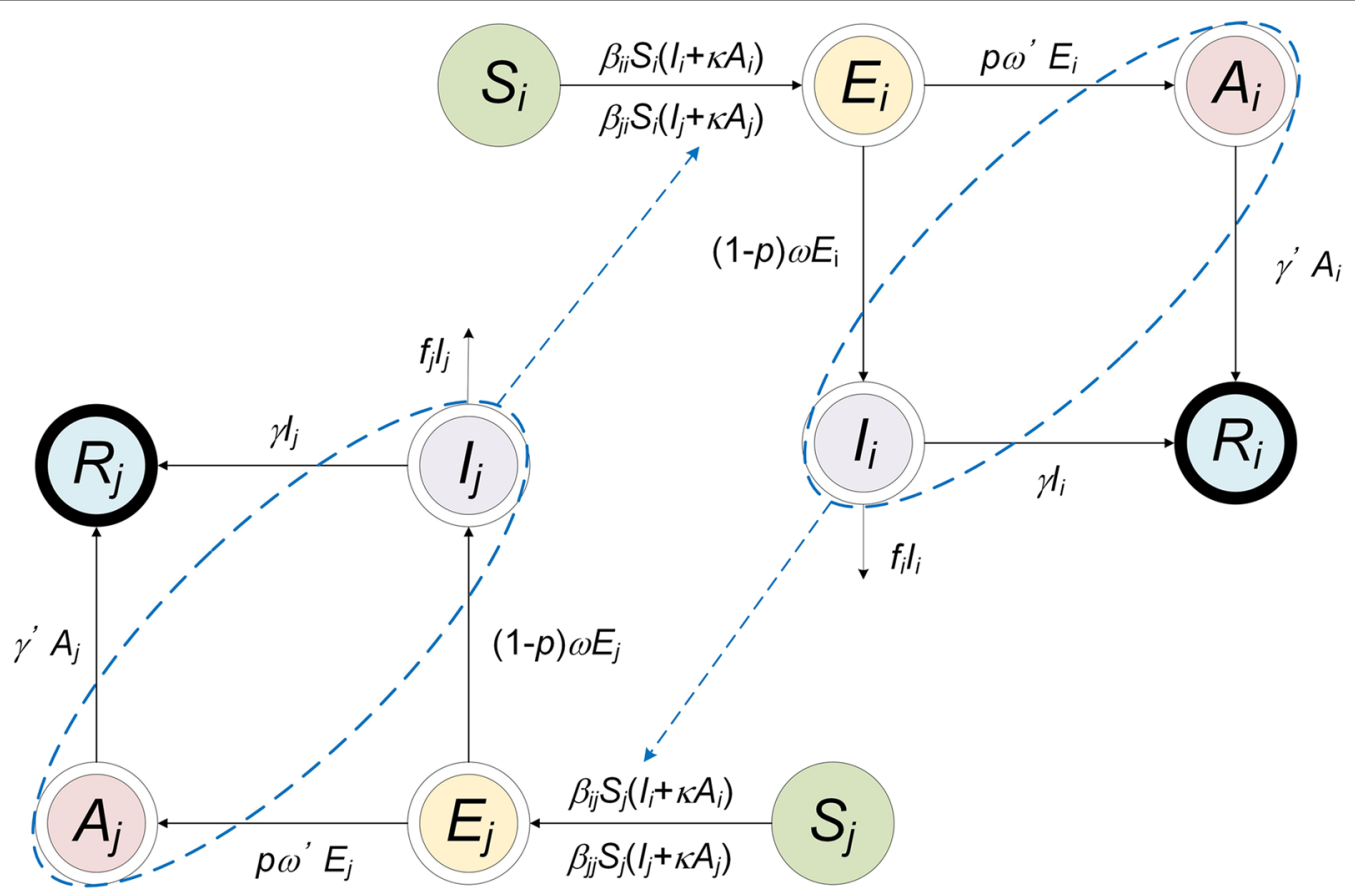

Fig. 2 The flowchart of the age-specific SEIAR model (Model 1) 


$$
\begin{aligned}
\frac{d I_{i}}{d t}= & (1-p) \omega E_{i}-\gamma I_{i}-f_{i} I_{i} \\
\frac{d A_{i}}{d t}= & p \omega^{\prime} E_{i}-\gamma^{\prime} A_{i} \\
\frac{d R_{i}}{d t}= & \gamma I_{i}+\gamma^{\prime} A_{i} \\
\frac{d V_{1 i}}{d t}= & \delta_{i} S_{i}-\beta_{i i} V_{1 i}\left(I_{i}+\kappa A_{i}\right)-\beta_{j i} V_{1 i}\left(I_{j}+\kappa A_{j}\right)-\varphi V_{1 i} \\
\frac{d V_{2 i}}{d t}= & \varphi V_{1 i}-(1-\lambda) \beta_{i i} V_{2 i}\left(I_{i}+\kappa A_{i}\right) \\
& -(1-\lambda) \beta_{j i} V_{2 i}\left(I_{j}+\kappa A_{j}\right) \\
\frac{d S_{j}}{d t}= & -\beta_{j j} S_{j}\left(I_{j}+\kappa A_{j}\right)-\beta_{j i} S_{j}\left(I_{i}+\kappa A_{i}\right)-\delta S_{j} \\
\frac{d E_{j}}{d t}= & \beta_{j j}\left(V_{1 j}+(1-\lambda) V_{2 j}+S_{j}\right)\left(I_{j}+\kappa A_{j}\right)+\beta_{j i}\left(V_{1 j}\right. \\
& \left.+(1-\lambda) V_{2 j}+S_{j}\right)\left(I_{i}+\kappa A_{i}\right)-(1-p) \omega E_{j}-p \omega^{\prime} E_{j}
\end{aligned}
$$$$
\frac{d I_{j}}{d t}=(1-p) \omega E_{j}-\gamma I_{j}-f_{i} I_{j}
$$$$
\frac{d A_{j}}{d t}=p \omega^{\prime} E_{j}-\gamma^{\prime} A_{j}
$$$$
\frac{d R_{j}}{d t}=\gamma I_{j}+\gamma^{\prime} A_{j}
$$$$
\frac{d V_{1 j}}{d t}=\delta_{j} S_{j}-\beta_{i j} V_{1 j}\left(I_{j}+\kappa A_{j}\right)-\beta_{i j} V_{1 j}\left(I_{i}+\kappa A_{i}\right)-\varphi V_{1 j}
$$$$
\begin{aligned}
\frac{d V_{2 j}}{d t}= & \varphi V_{1 j}-(1-\lambda) \beta_{j j} V_{2 j}\left(I_{j}+\kappa A_{j}\right) \\
& -(1-\lambda) \beta_{i j} V_{2 j}\left(I_{i}+\kappa A_{i}\right)
\end{aligned}
$$$$
N=S_{i}+E_{i}+I_{i}+A_{i}+R_{i}+V_{1 i}+V_{2 i}
$$

The left side of the differential equation shows the instantaneous change rates of $S, E, I, A$, and $R$, at time $t$. The subscripts $i$ and $j(i \neq j)$ represent age groups 1-4.

In the age-specific SEIAR model, two compartments were added, which were defined as vaccinated individuals without immunity $\left(V_{1}\right)$ and vaccinated individuals with immunity $\left(V_{2}\right)$. We built two models (Model 2 and Model 3) based on the following assumptions:
A flowchart of Model 2 is shown in Fig. 3. The following assumption should be added to the age-specific SEIAR model:

(a) Assuming that only susceptible individuals were vaccinated, $\delta$ is considered the daily vaccination rate.

(b) Vaccinated individuals without immunity, infected by contact with symptomatic or asymptomatic cases; the relative transmission rate is also $\beta_{i i}$ within the age group and $\beta_{i j}$ between age groups.

(c) Vaccinated individuals without immunity will become immune after a period. Protective antibodies will occur within $1 / \phi$ days, which is described as the period in which vaccinated individuals without immunity will become immune.

(d) The COVID-19 vaccine has a VE defined as $\lambda$. Vaccinated individuals with immunity are infected by contact with symptomatic or asymptomatic patients. The infection rate is defined as $1-\lambda$.

The equations used in the model (Model 2) are in Additional file 2: Text S1.

We assumed that the VE of COVID-19 was similar to what was previously reported for the $\mathrm{H} 1 \mathrm{~N} 1$ pandemic [18] (Model 3), which quantified the different protective effects as follows: $\mathrm{VE}_{S}$ refers to $\mathrm{VE}$ against susceptibility, $\mathrm{VE}_{I}$ as $\mathrm{VE}$ against infectiousness, and $\mathrm{VE}_{P}$ as $\mathrm{VE}$ against pathogenicity or symptomatic illness has given infection. In addition, according to the theory of herd immunity [19], we have considered the coefficient of herd immunity $(\theta)$, defined as the proportion of herd immunity, which was calculated as follows:

$$
\theta=1-\frac{\text { Total number of new cases }}{N}
$$

Here, we added three parameters into the model, including $x$ referring to a decreased proportion of VE against susceptibility, $y$ referring to as a decreasing proportion of VE against infectivity, and $z$ referring to as a decreasing proportion of VE against pathogenicity. The equations used for calculation are as follows:

$$
\begin{aligned}
& x=1-V E_{S} \\
& y=1-V E_{I} \\
& z=1-V E_{p}
\end{aligned}
$$

A flowchart of Model 3 is presented in Fig. 4. The following assumption should be added to the age-specific SEIAR model: 


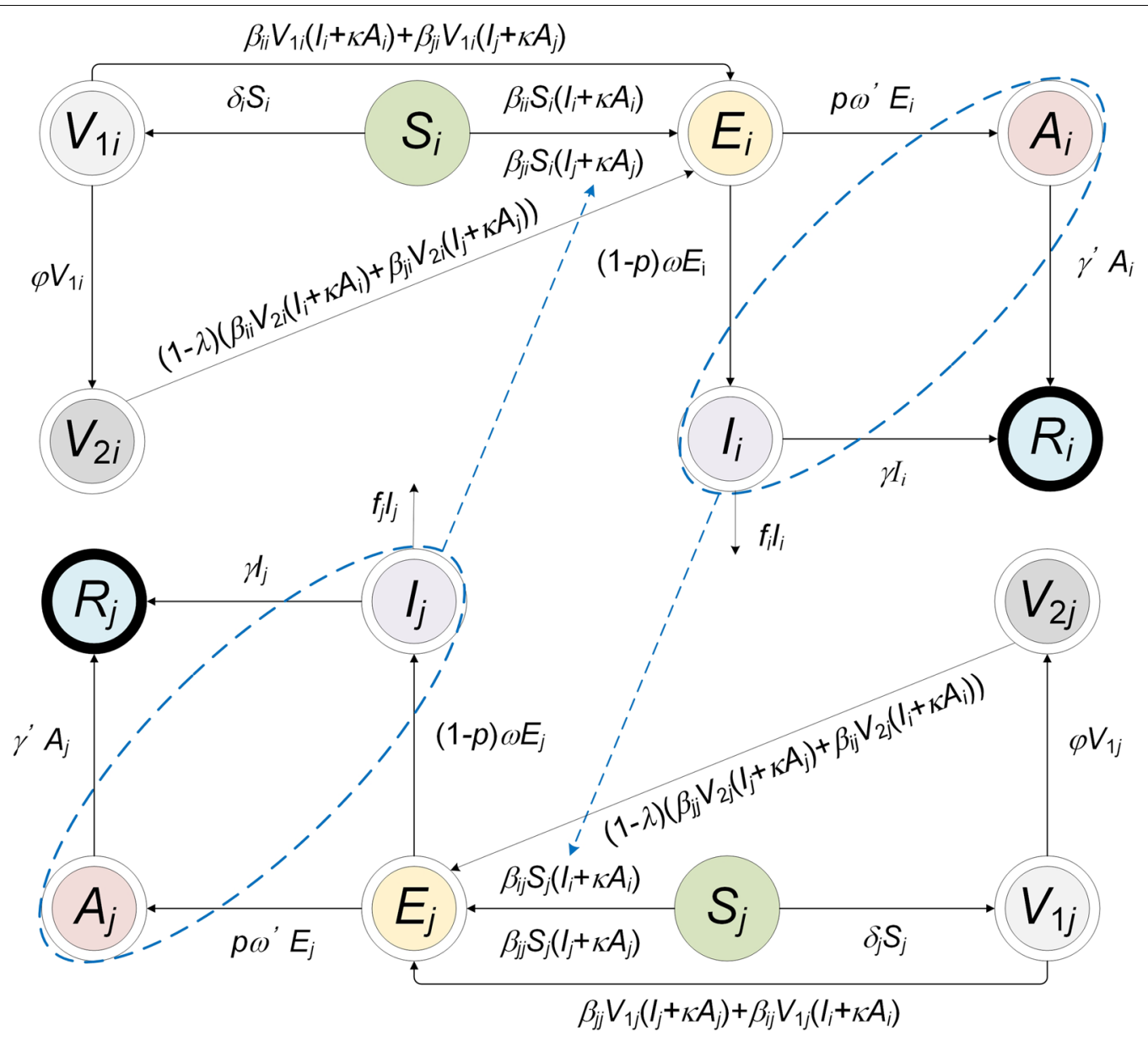

Fig. 3 The flowchart of vaccinated age-specific SEIAR model (Model 2). $i$ and $j$ represent age $\leq 14,15-44,45-64$, and $\geq 65$, respectively $(i \neq j$ )

(a) Once herd immunity has been attained, susceptible individuals are infected following contact with symptomatic or asymptomatic patients. The proportion of herd immunity is $\theta$.

(b) Vaccinated individuals without immunity $\left(V_{1}\right)$ would be infected by contact with two types of cases, namely, non-vaccinated symptomatic/ asymptomatic cases and vaccinated symptomatic/ asymptomatic cases. Meanwhile, the infectivity of $I_{2}$ and $A_{2}$ would decrease by a proportion of $y$.

(c) Vaccinated individuals with immunity $\left(V_{2}\right)$ would be infected following contact with two types of cases, namely, non-vaccinated symptomatic/ asymptomatic cases and vaccinated symptomatic/ asymptomatic cases. The susceptibility of $V_{2}$ would be decreased by a proportion of $x$ and the pathogenicity of SARS-CoV-2 would decrease by a proportion of $z$.

The equations used in the model (Model 3) are Additional file 2: Text S1.

\section{Vaccination simulation scenarios}

In this study, we simulated the vaccine's effects based on the stage before travel ban in Wuhan City (stage 1). We developed two scenarios (referred to as scenario I and scenario II) to assess the vaccine's effects (Fig. 5). Thereafter, we built 19 sub-scenarios (defined as scenes) based on the above two scenarios. The conditions of the scenarios were as follows:

(a) Scenario I: According to Model 2, we assumed that susceptible people could be vaccinated at a rate of $\delta$ and achieve immunity after $1 / \phi$ days. Previously immune individuals could be infected at a rate of $1-\lambda$. We assumed the VE of COVID-19 is similar to that of measles and influenza vaccines and simulates effects by changing the parameters $\delta$, $\phi$, and $\lambda$. The conditions of the 11 scenes (I to XI) are shown in Additional file 3: Table S2.

(b) Scenario II: Model 3 also assumes that susceptible people could be vaccinated at a rate of $\delta$ and achieve immunity after $1 / \phi$ days. Furthermore, we assumed 


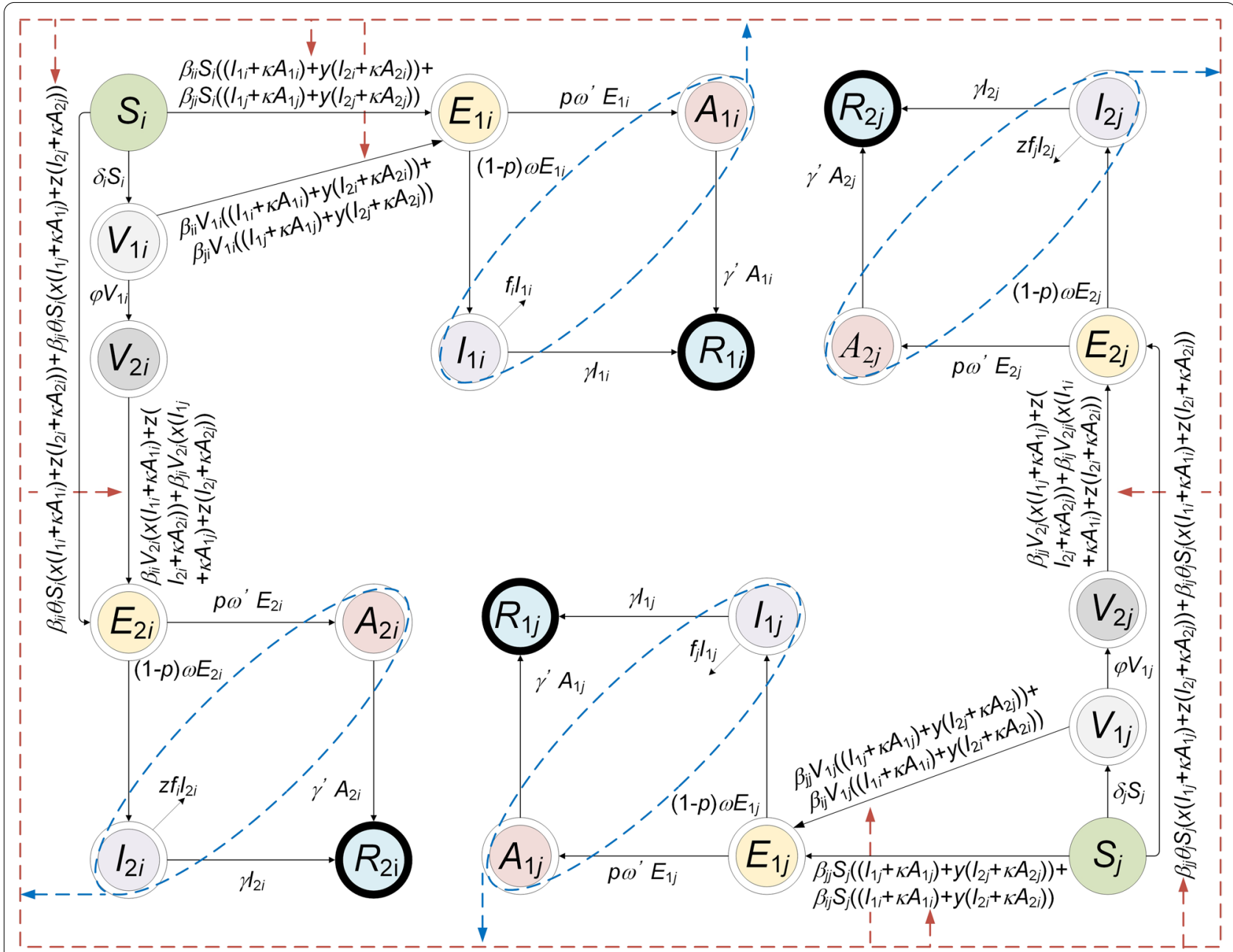

Fig. 4 The flowchart of vaccinated age-specific SEIAR model (Model 3, considering the vaccine efficacy of susceptibility and infectivity). $i$ and $j$ represent age $\leq 14,15-44,45-64$, and $\geq 65$, respectively $(i \neq j)$

that if immune people become ill, infectivity could be reduced after vaccination; the remaining ratio after reduction is $y$. Susceptibility could be reduced after vaccination if immune people contacted disease cases, and the remaining ratio after reduction was $x$. If contact occurred between immune people and cases, both parameters could be reduced. The future vaccine has similar effects as influenza A (H1N1) and simulated vaccine effects by changing the parameters $\delta, \mathrm{x}, \mathrm{y}$, and $z$. The total population was divided into two groups, vaccinated and nonvaccinated. The conditions of the eight scenes (XII to XIX) are shown in Additional file 3: Table S2.

Additionally, we assumed that the severity of disease would be decreased after vaccination, and the coefficient was the same as the parameter $z=0.33$ (decreasing the proportion of VE) in scenes XIV-XVII of scenario II.

\section{Parameter estimation}

In this study, several parameters were adopted to develop the model, and the description, value, and method were listed in Table 2. The parameter $\kappa$ refers to the relative transmissibility rate of asymptomatic to symptomatic individuals. In the model, $\kappa$ is set to 0.65 . The most important reason is that a study reported that $4.11 \%$ of individuals would become infected after close contact with asymptomatic patients, versus $6.3 \%$ for individuals infected after close contact with symptomatic patients [20]. In addition, it has been reported that the transmissibility of symptomatic patients is 3.9 times that of asymptomatic patients, and an asymptomatic individual 


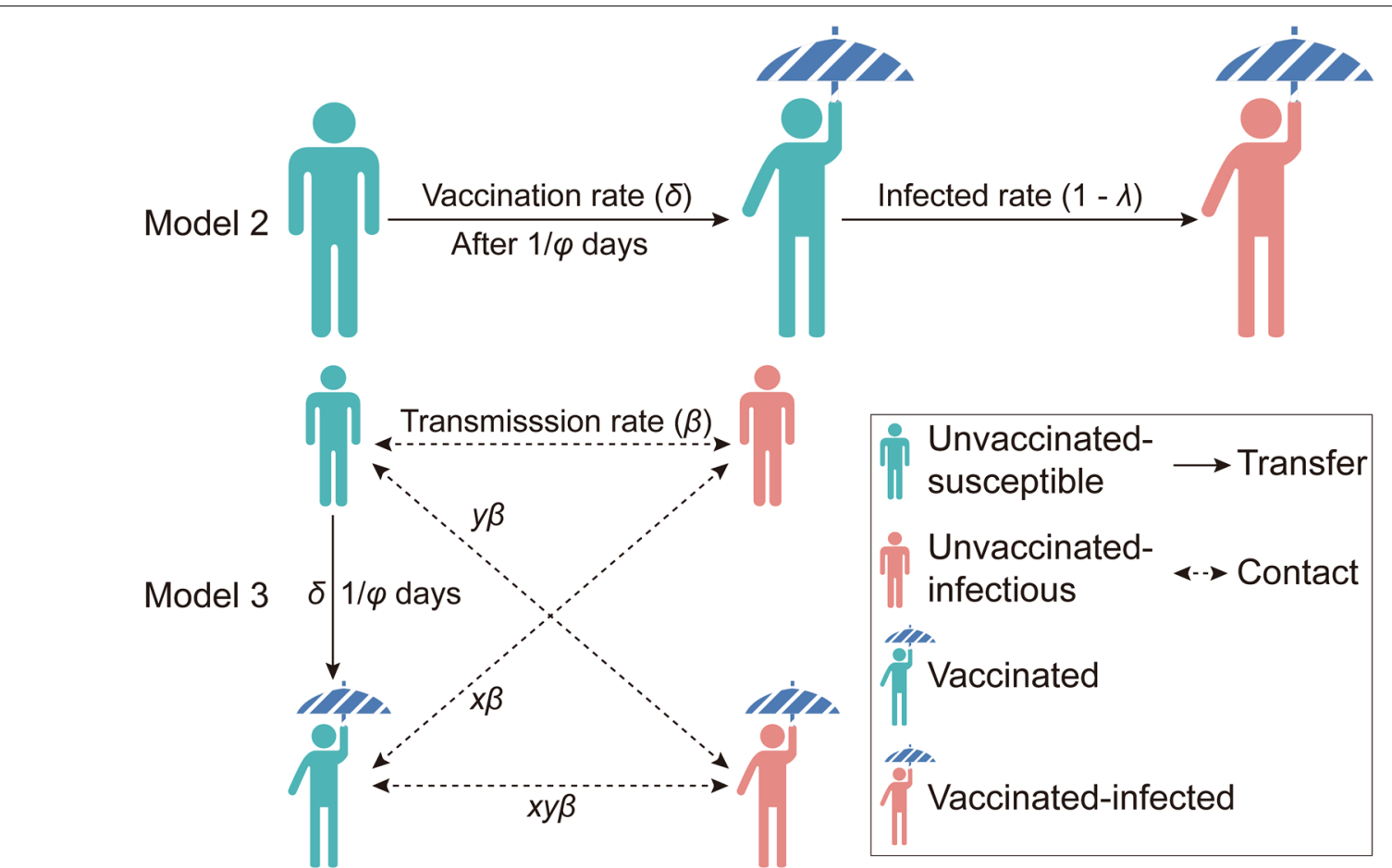

Fig. 5 The process of vaccination in Model 2 and Model 3. Parameter $\delta, \lambda, \beta, \varphi, x$ and $y$ represent vaccination rate, vaccine efficacy, transmission rate, immune relative rate, decreasing proportion of vaccine efficacy against susceptibility, and decreasing proportion of vaccine efficacy against infectivity, respectively

may contribute to 11 infectious cases [21, 22]. In their dynamic model, the parameter $\kappa$ was set to 1.0 [12].

Several parameters are summarized in Fig. 6. The proportion of asymptomatic cases in the Diamond Princess cruise ship was $17.9 \%$ (95\% confidence interval $[C I]$ : 15.5-20.2\%) and a study estimated the asymptomatic ratio as $30.8 \%$ (95\% CI: 7.7-53.8\%), by binomial distribution [23, 24]. Meanwhile, the asymptomatic proportion was reportedly $20.75 \%$ in Ningbo City, while another study indicated that it was much greater $(78 \%)$. Therefore, we set the asymptomatic proportion $(p)$ to 0.36 in the model (Fig. 6A).

According to published research, the incubation period during the early epidemic was 4 days (interquartile range: $2-7)$ in Wuhan City [25] and 5.1 days (95\% CI: 4.5-5.8), based on publicly reported data [26]. Still, there is a wide range of incubation periods reported including incubation period in Ningbo City (2-18) and 95\% CI in Wuhan City (2-14) [20,27]. In the model, the incubation period was set to 5 days $\left(\omega=\omega^{\prime}=0.2\right)$.

In this study, it was assumed that the diagnosed cases would be immediately removed from the population. In the epidemiological characteristics of COVID-19 in Hong Kong, the period from symptom onset to confirmed diagnosis was 6.39 days (range: $1-8.83$ ) [28]. The right truncation data indicated that the time from illness onset to hospitalization ranged from 2.7 to 8 days [27]. Meanwhile, other studies reported various findings regarding the time from symptom onset to hospitalization was, such as 7 days, 4-6 days, and 4.1-7.5 days [2931]. In this model (Fig. 6C), the infectious period was set to 5 days $(\gamma=0.2)$. However, another study indicated that the median communicable period of 24 asymptomatic cases was 9.5 (range: $1-21$ ) days [32]. We set $\gamma^{\prime}$ to 0.1 in the current model.

In Wuhan City, the total population was set to 11 080996 ( $\leq 14$ years: 1256 552; 15-44 years: 5210 885; 45-64 years: 3 374 388; $\geq 65$ years: 1239 171) for modelling purposes; according to the data analyses, the case fatality rate $(f)$ was set to $0.1681 \%$ for age group 1 , $0.5490 \%$ for age group 2, 3.4168\% for age group 3, and $14.8424 \%$ for age group 4 .

In the model, the vaccination rate $(\delta)$ was set to 0.00001, 0.0001, 0.001, $0.010 .02,0.03,0.04,0.05,0.06$, $0.07,0.08,0.09$, and 0.1 based on the assumption of vaccination priority. In addition, we assumed that the effects of the COVID-19 vaccine would be similar to those of influenza $\mathrm{A}$, influenza $\mathrm{B}$, or measles vaccine in the scenario I. Most studies indicated that the time of development of influenza immunity ranged between 7 and 
Table 2 Description and source of parameters in the age-specific model

\begin{tabular}{|c|c|c|c|c|c|}
\hline Parameter & Description & Unit & Value & Range & Method \\
\hline$\beta_{i i}{ }^{*}$ & Transmission relative rate among age group $i$ & Individuals ${ }^{-1} \cdot$ days $^{-1}$ & - & $\geq 0$ & Curve fitting \\
\hline$\beta_{i j}{ }^{*}$ & Transmission relative rate from age group $i$ to $j$ & Individuals ${ }^{-1} \cdot$ days $^{-1}$ & - & $\geq 0$ & Curve fitting \\
\hline$\beta_{j i}{ }^{*}$ & Transmission relative rate from age group $j$ to $i$ & Individuals ${ }^{-1} \cdot$ days $^{-1}$ & - & $\geq 0$ & Curve fitting \\
\hline$\beta_{j j}{ }^{*}$ & Transmission relative rate among age group $i$ & Individuals ${ }^{-1} \cdot$ days $^{-1}$ & - & $\geq 0$ & Curve fitting \\
\hline k & Relative transmissibility rate of asymptomatic to symptomatic individuals & 1 & 0.65 & $0-1$ & {$[12,21,22]$} \\
\hline$p$ & Proportion of the asymptomatic & 1 & 0.36 & $0.016-0.78$ & {$[23,24]$} \\
\hline$\omega$ & Incubation relative rate & Days $^{-1}$ & 0.2 & $0.05556-0.5$ & {$[20,25-27]$} \\
\hline$\omega^{\prime}$ & Latent relative rate & Days $^{-1}$ & 0.2 & $0.05556-0.5$ & {$[20,25-27]$} \\
\hline y & Recovered/Removed rate of the infectious & Days $^{-1}$ & 0.2 & $0.1111-0.3333$ & {$[27-31]$} \\
\hline$\gamma^{\prime}$ & Recovered/Removed rate of the asymptomatic & Days $^{-1}$ & 0.1 & $0.04762-1$ & {$[32]$} \\
\hline$f_{i}$ & Fatality of the disease of age group $i$ & 1 & - & $0-1$ & Analysis of data \\
\hline$\delta$ & Vaccination rate & Days $^{-1}$ & - & $0-1$ & Assumption \\
\hline$\varphi$ & Immune relative rate & 1 & - & $0-1$ & {$[33-37]$} \\
\hline$\lambda$ & Vaccine efficacy & 1 & - & $0-1$ & {$[38-50]$} \\
\hline$\theta$ & Proportion of herd immunity & 1 & 0 & $0-1$ & Assumption \\
\hline$x$ & Decreasing proportion of vaccine efficacy against susceptibility & 1 & 0.6 & $0-1$ & [18] \\
\hline y & Decreasing proportion of vaccine efficacy against infectivity & 1 & 0.6 & $0-1$ & [18] \\
\hline$z$ & Decreasing proportion of vaccine efficacy against pathogenicity & 1 & 0.33 & $0-1$ & [18] \\
\hline
\end{tabular}

- Not applicable

14 days [33-35]. Meanwhile, the highest rate of immunoglobulin $G$ positivity in measles occurred in weeks 4 and 5 post-vaccination [36,37]. In the model, immunity was set to 30 days after measles vaccination. Therefore, according to the effect of influenza $\mathrm{A}$, influenza $\mathrm{B}$, and measles, we set the immune relative rate $(\phi)$ to $1 / 7,1 / 14$, and $1 / 30$, respectively. In addition, we simulated a scene to explore the conditions in which $\phi$ was equal to $1 / 5$, $1 / 15,1 / 20,1 / 25$, and $1 / 30$, respectively. Several studies have reported that the VE of influenza ranged from 19 to $91 \%$, with an especially wide range for influenza A (Fig. 6D) [38-42]. In contrast, the VE of measles was reported to range from 45 to $99 \%$, and the median was approximately $90 \%$ (Fig. 6-E) [43-50]. In this study, VE $(\lambda)$ was set to $0.3,0.7$, and 0.9. Meanwhile, a scene based on different VE was simulated in the model, set $\lambda$ to $0,0.1,0.2$, ..., 0.9, and 1. Thereafter, the parameters of scenario II were collected from a study that indicated $\mathrm{VE}_{S}=0.4$, $\mathrm{VE}_{I}=0.4$, and $\mathrm{VE}_{P}=0.67$. In the model, $x=0.6, y=0.6$, and $z=0.33$. Meanwhile, the immune relative rate $(\phi)$ was set to 0.1 in scenario II, according to the vaccine effect of H1N1.

Most studies have reported that the mean value of VE was more than $90 \%$ (with two-dose administration) for controlling ancestral virus (Fig. 6-F) [5-7, 9, 51], 68\%-95\% (with two-dose administration) for controlling infection of the Alpha variant, and more than 62\% (with two-dose administration) for controlling infection of the Delta variant (Fig. 6G) [8].

\section{Estimated transmission}

In this study, we adopted two indicators to estimate the transmissibility and risk of infection, including the effective reproduction number $\left(R_{\mathrm{eff}}\right.$, the mean number of secondary cases an infected person can cause in a population after implementation of intervention measures), and the probability of infection from a single contact ( $q$, the possibility of the susceptible person becoming infected after effective contact).

The equation of $R_{\text {eff }}$ is as follows:

$$
R_{\mathrm{eff}}=\beta N\left(\frac{1-p}{\gamma}+\frac{\kappa p}{\gamma^{\prime}}\right)
$$

The equation of $q$ is as follows:

$$
\beta N=1-(1-q)^{\alpha}
$$

In the above equation, $\alpha$ is defined as the contact frequency per day, which was calculated from a previously published paper [52].

To compare the transmission in different areas, we calculated the median of the normalized $R_{\mathrm{eff}}$ in four stages. The relative transmissibility in the different age groups was quantified by an equation, and the min-max normalized (the lower and upper bounds of relative transmissibility) version was used:

$$
\text { Normalized } R_{\mathrm{eff}}=\frac{x-\min (x)}{\max (x)-\min (x)}
$$



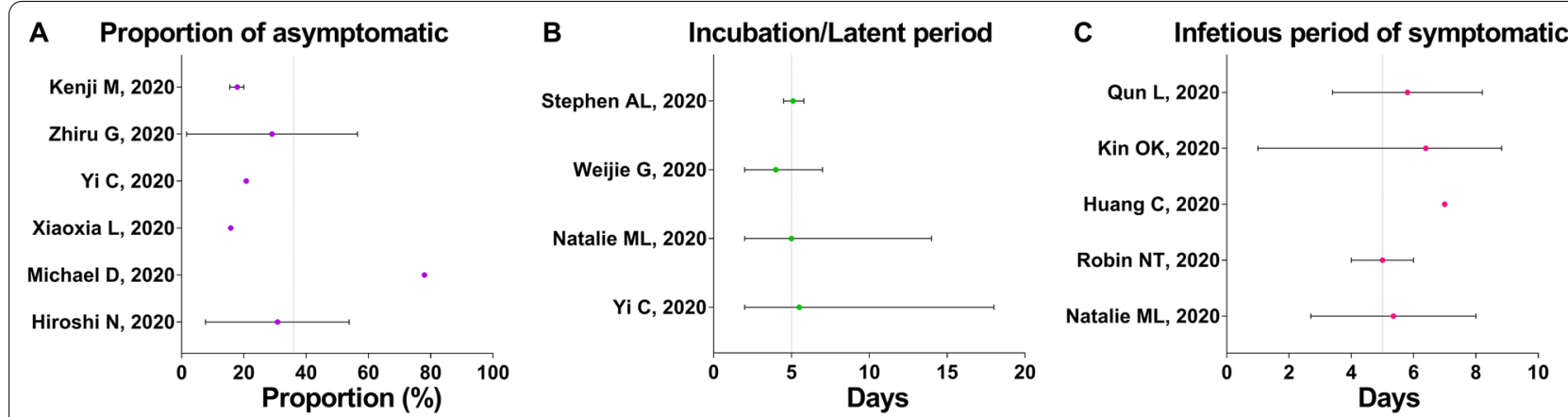

D

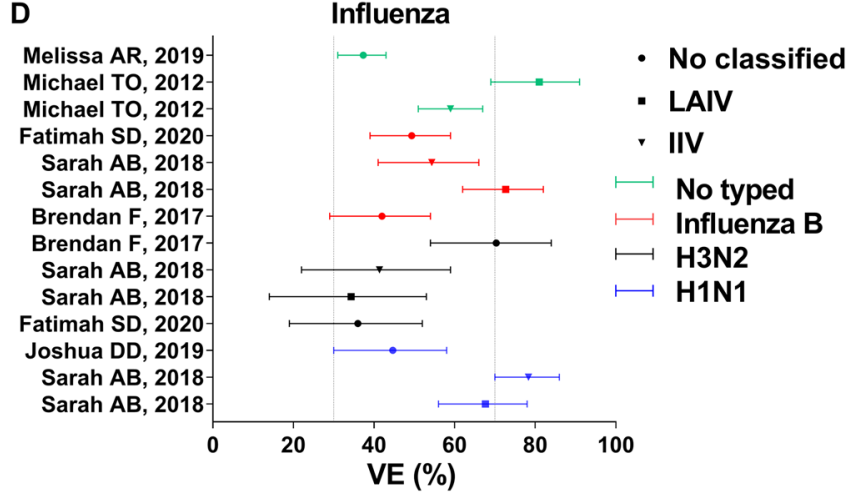

$\mathbf{F}$

Ancestral virus

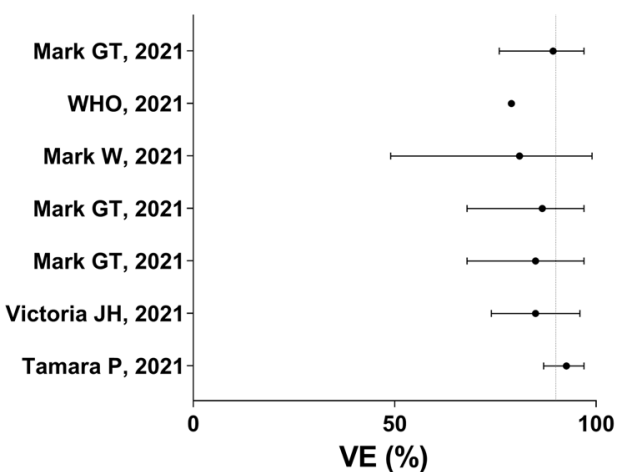

$\mathbf{E}$

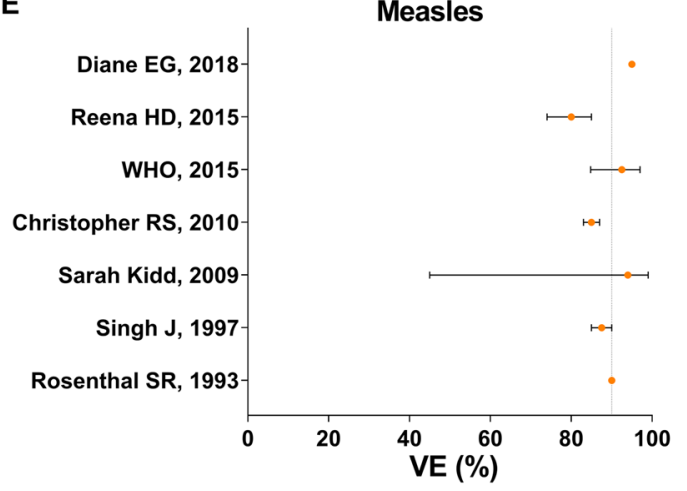

G

Variants

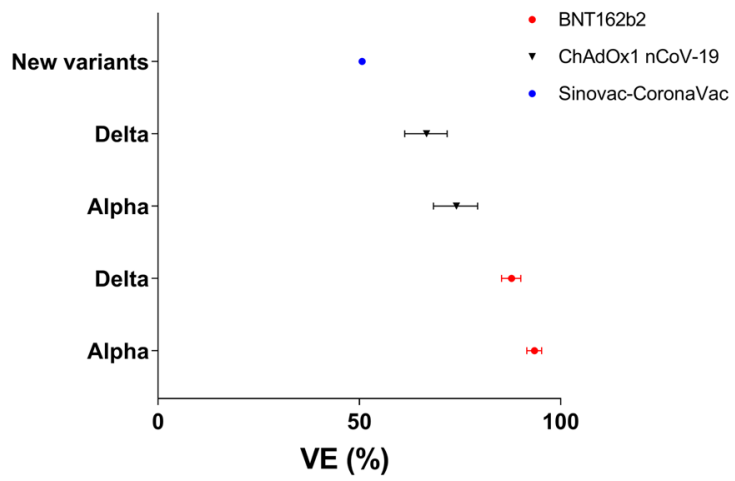

Fig. 6 Summary of reported parameters of model about natural history and vaccination. A Proportion of asymptomatic $(p)$. B Incubation period $(\omega)$ and latent period $\left(\omega^{\prime}\right)$. C Infectious period of symptomatic $(\gamma)$. D VE of influenza. $\mathbf{E} \vee E$ of measles. $\mathbf{F} \vee E$ after two doses vaccinating for the ancestral virus of COVID-19. G VE after two doses vaccinating for new variants of COVID-19

$x$ is the value of $R_{\text {eff }-i j}$ (subscript $i$ and $j(i \neq j)$ equals 1 to 4 , respectively). Thereafter, we further compared the normalized results of Wuhan City, Hunan Province, and Jilin Province to explain the heterogeneity of age-related transmission in different areas. The results of the above provinces were from a previously published paper [12]; we re-calculated $R_{\text {eff }}$ according to the parameter $\beta$ results.

To explore transmission interactions (infected and infected by others) in the four age groups, we adopted two indicators to estimate the infectivity and susceptibility of each group in four stages. $R_{\mathrm{I}}$ indicated infectivity, and $R_{\mathrm{s}}$ was the susceptibility in a specific age group, which was calculated as follows:

$$
\begin{aligned}
& R_{\mathrm{I}-i}=\sum_{j=1}^{n} R_{\mathrm{eff}-i j} \\
& R_{\mathrm{S}-i}=\sum_{j=1}^{n} R_{\mathrm{eff}-j i}
\end{aligned}
$$


In the above equation, $n$ is equal to four. For example, the $R_{\mathrm{I}}$ of age group 1 is the sum of $R_{\text {eff-11 }}, R_{\text {eff- } 12}, R_{\text {eff- } 13}$, and $R_{\text {eff-14 }}$, and the $R_{\mathrm{S}}$ of age group 1 is the sum of $R_{\text {eff-11 }}$, $R_{\text {eff-21 }}, R_{\text {eff-31 }}$, and $R_{\text {eff-41 }}$.

\section{Vaccine effectiveness}

We evaluated the vaccination effects for controlling transmission and disease severity.

The transmission was estimated using six indicators, including the total number of new cases $(T N)$, total attack rate $(T A R)$, and the number of new cases at peak $(N P)$, and two positive indicators, duration of outbreak $(D O)$ and peak time $(P T)$. The equation is as follows:

$$
\begin{aligned}
& T N=\text { Total number of new cases } \\
& T A R=\frac{T N}{N} \times 100 \% \\
& D O=t_{0}-t_{1} \\
& P T=t_{p} \\
& N P=\text { Number of new cases at peak }
\end{aligned}
$$

In the above equation, $N, t_{0}, t_{1}$, and $t_{p}$ refer to the number of total population, illness onset date of the first case, illness onset date of the last case, and the peak of the infection curve, respectively.

The disease severity was estimated based on the total number of deaths $(N D)$. The equation is as follows:

$$
N D=\text { Total number of death }
$$

Because of the competitive relationship between vaccination rate $(\delta)$ and the relative transmission relative $(\beta)$, we cannot directly consider vaccination rate as vaccination coverage. In the model, we assumed that the start time of vaccination was $t_{s}$, and the end time (the vaccination time of the last susceptible person) was $t_{e}$. We calculated the vaccination coverage for the total population and each age group. The vaccination coverage for each day was calculated from the differential equation of the vaccination model. Model 2 was calculated as follows:

$$
\operatorname{Vaccination} \text { coverage }(n)=\frac{\delta_{i}}{\beta_{i i}\left(I_{i}+\kappa A_{i}\right)+\beta_{j i}\left(I_{j}+\kappa A_{j}\right)+\delta_{i}}
$$

Model 3 was calculated as follows:

$$
\begin{aligned}
\text { Vaccination coverage }(n)= & \delta_{i} /\left(\beta_{i i}\left(\left(I_{1 i}+\kappa A_{1 i}\right)+y\left(I_{2 i}+\kappa A_{2 i}\right)\right)\right. \\
& +\beta_{j i}\left(\left(I_{1 j}+\kappa A_{1 j}\right)+y\left(I_{2 j}+\kappa A_{2 j}\right)\right) \\
& +\beta_{i i} \theta_{i}\left(x\left(I_{1 i}+\kappa A_{1 i}\right)+z\left(I_{2 i}+\kappa A_{2 i}\right)\right) \\
& \left.+\beta_{j i} \theta_{i}\left(x\left(I_{1 j}+\kappa A_{1 j}\right)+z\left(I_{2 j}+\kappa A_{2 j}\right)\right)+\delta_{i}\right)
\end{aligned}
$$

$n$ refers to vaccination coverage of $n$th day. Thereafter, the total vaccination coverage was calculated by integrating vaccination coverage of the vaccination period (from $t_{s}$ to $\left.t_{e}\right)$. The equation is as follows:

$$
\text { Total vaccination coverage }=\int_{t_{s}}^{t_{e}} \text { vaccination coverage }(t) d t
$$

In the equation, $t_{s}$ and $t_{e}$ refer to the start time of the vaccination period and the end time (the vaccination time of the last susceptible person), respectively.

\section{Simulation and statistical analysis}

According to previously published studies [53-55], we assumed that heterogeneity of transmissibility existed as an ascending trend and then a descending trend. The data were divided into several segments (defined as stages); for example, Wuhan City was divided into four stages in the disease transmission period (Fig. 7). The period of each stage was as follows:

(a) Stage 1 refers to the illness onset date from December 2, 2019, to January 23, 2020.

(b) Stage 2 refers to the illness onset date from January 24 to February 2, 2020.

(c) Stage 3 refers to the illness onset date from February 3 to February 18, 2020.

(d) Stage 4 refers to the illness onset date from February 19, 2020, to March 16, 2020.

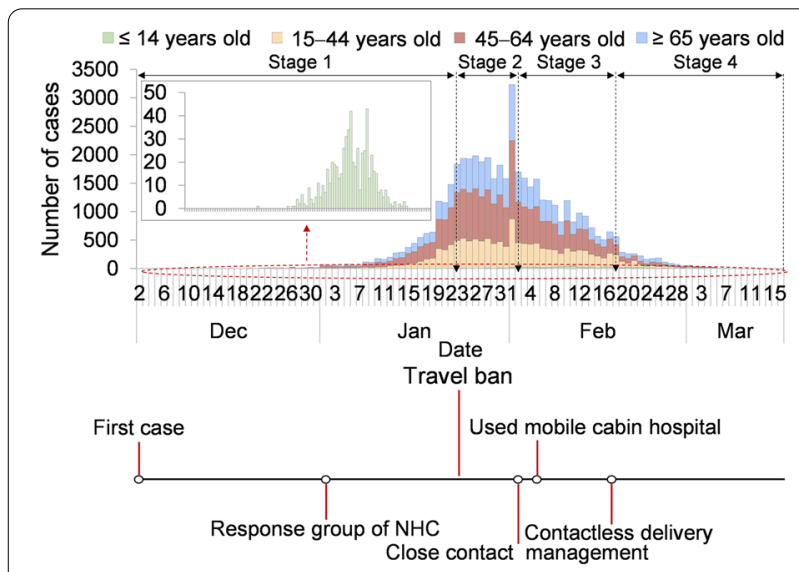

Fig. 7 Epidemic curve of four age-group and key interventions in Wuhan City. A Epidemic curve. B Intervention measures 
Curve fitting and model simulation adopted the least root-mean-square deviations. The simulation method used the Runge-Kutta method of order four with a tolerance set to 0.001 . The differential equations were solved in steps of 0.02 days. The goodness of fit was judged by the coefficient of determination $\left(R^{2}\right)$.

\section{Results}

\section{Epidemiological characteristics and model effectiveness}

From December 2, 2019, to March 16, 2020, a total of 47722 new cases (cumulative incidence: 431 cases per 10000 persons) were reported in Wuhan City (Fig. 7). In those $\leq 14$ years old, there were 595 reported cases (cumulative incidence: 47 cases per 10000 persons); 12 933 cases were reported in 15-44-year-olds (cumulative incidence: 248 cases per 10000 persons), 20,106 cases reported among 45-64-year-olds (cumulative incidence: 596 cases per 10000 persons) and 14,088 cases reported among those $\geq 65$ years old (cumulative incidence: 1134 cases per 10000 persons).

The date of the first case onset was on December 2, 2019. The National Health Commission of China set up a response group on January 1, 2020. Thereafter, several intervention measures were adopted by Wuhan City, such as travel ban (January 23, 2020), close-contact management (February 3, 2020), mobile cabin hospitals (February 5, 2020), and contactless delivery (February 17, 2020).

In Wuhan City, the age-specific model (Fig. 8) fits the total reported data for the four age groups most effectively $\left(\leq 14\right.$ years: $R^{2}=0.823, P<0.0001 ; 15-44$ years: $R^{2}=0.944, \quad P<0.0001 ; \quad 45-64 \quad$ years: $R^{2}=0.948$, $P<0.0001 ; \geq 65$ years: $\left.R^{2}=0.940, P<0.0001\right)$. However, the model fits the reported data in stage 2 and is not significant in stage 3 in those $\leq 14$ years of age.

\section{Transmissibility and risk of infection}

According to the simulated results (Fig. 9), in stage 1, the highest transmissibility occurred among the members of age group $2\left(\beta_{22}=1.50 \times 10^{-7}, R_{\text {eff- } 22}=4.28\right)$, followed by age group 2 to $3\left(\beta_{23}=1.57 \times 10^{-7}, R_{\text {eff- } 23}=2.61\right)$, age group 2 to $4\left(\beta_{24}=3.26 \times 10^{-7}, R_{\text {eff- } 24}=1.69\right)$, among the members of group $3\left(\beta_{33}=8.43 \times 10^{-8}, R_{\text {eff- } 33}=1.44\right)$, and age group 4 to $3\left(\beta_{43}=8.39 \times 10^{-8}, R_{\text {eff- } 43}=1.44\right)$. In stage 2 , the highest transmissibility occurred from age group 2 to $4\left(\beta_{24}=2.12 \times 10^{-7}, R_{\text {eff- } 24}=1.10\right)$, while the other group combinations had a $R_{\text {eff }}$ value lower than 1 . Thereafter, all the values of $R_{\text {eff }}$ were lower than 1 in stages 3 and 4. Our results suggest that transmission was effectively controlled after travel ban in Wuhan City.

In stage 1 , the highest infectivity was in age group 2 $\left(R_{\mathrm{I}}=8.58\right)$, followed by age group $4\left(R_{\mathrm{I}}=3.58\right)$, age group
$3\left(R_{\mathrm{I}}=2.58\right)$, and age group $1\left(R_{\mathrm{I}}=1.62\right)$. The highest susceptibility was observed in age group $3\left(R_{\mathrm{S}}=6.44\right)$, followed by age group $2\left(R_{\mathrm{S}}=6.14\right)$, age group $4\left(R_{\mathrm{S}}=3.75\right)$, and age group $1\left(R_{\mathrm{S}}=0.03\right)$. In stage 2 , the infectivity and susceptibility were as follows: in age group $1\left(R_{\mathrm{I}}=1.10\right.$, $\left.R_{\mathrm{S}}=0.04\right)$, age group $2\left(R_{\mathrm{I}}=2.45, R_{\mathrm{S}}=2.42\right)$, age group $3\left(R_{\mathrm{I}}=1.42, R_{\mathrm{S}}=2.10\right)$, and age group $4\left(R_{\mathrm{I}}=1.49\right.$, $\left.R_{\mathrm{S}}=1.07\right)$, respectively. The values of $R_{\mathrm{I}}$ and $R_{\mathrm{S}}$ were all lower than 1 in stages 3 and 4 (Fig. 10). In addition, susceptibility tended to increase in age group 1 from stage 1 to 3 (Fig. 11).

The highest contact frequency in stage 1 occurred among the members of age group $2(\alpha=53.32)$, followed by age group 2 to $3(\alpha=24.05)$, age group 2 to 1 $(\alpha=21.89)$, and among the members of age group 3 $(\alpha=15.82)$. In the elderly, we found the highest probability of infection to be from a single contact (Fig. 12). When considering the probability of infection from a single contact, the highest value in stage 1 occurred among the members of age group $2(q=15.144 \%)$, followed by age group 2 to 4 ( $q=6.647 \%$ ), age group 4 to 2 $(q=5.101 \%)$, and age group 3 to $4(q=4.797 \%)$. In stage 2 , the highest probability of infection from a single contact was occurred from age group 2 to $4(q=63.831 \%)$, followed by age group 1 to $4(q=22.763 \%)$, age group 1 to $3(q=17.410 \%)$, and age group 4 to $2(q=14.462 \%)$. In stage 3 , the highest probability of infection from a single contact was found in age group 1 to $4(q=16.177 \%)$, followed by age group 2 to 4 ( $q=5.779 \%)$, among the members of age group $4(q=5.728 \%)$, and age group 3 to 4 $(q=5.466 \%)$. In stage 4 , the highest value was found in age group 1 to 4 ( $q=0.397 \%)$, followed by age group 1 to $3(q=0.344 \%)$, age group 2 to $4(q=0.223 \%)$, and age group 4 to $3(q=0.217 \%)$.

We found differing age-specific transmission patterns in several areas (Fig. 13). The highest relative transmissibility in Wuhan City was from age group 2 to 4, followed by group 4 to 2,3 to 3, and 2 to 3 . In Hunan Province, the highest relative transmissibility was observed in age group 4 to 3, followed by group 3 to 4,2 to 2 , and 3 to 1 . In Jilin Province, it was observed from age group 4 to 4 , followed by group 3 to 4,2 to 2 , and 2 to 4 .

\section{Effectiveness of vaccination against transmission}

China should vaccinate at least $85 \%$ of the total population to interrupt transmission, with a VE of more than $70 \%$ (Figs. 14 and 15). The vaccine effects increased with an increase in vaccination rate (Fig. 16). However, it reached a threshold (Fig. 17) when $\delta_{i}=0.1$, regardless of scenario I $(T N=4722, T A R=0.04 \%, D O=177, P T=76$, $N P=68)$ or scenario II $(T N=4548464, T A R=41.05 \%$, $D O=997, P T=162, N P=56613)$. 


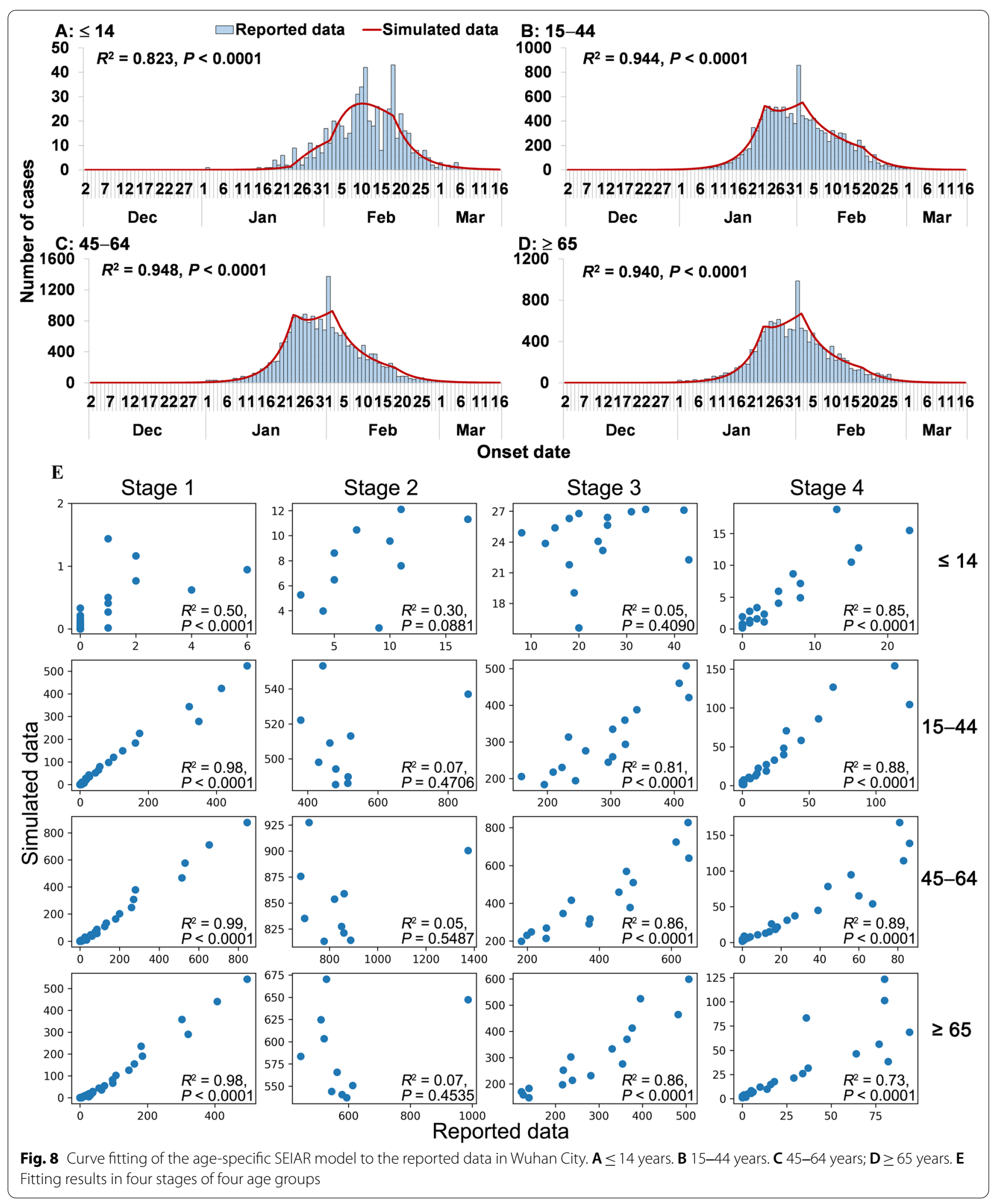




\section{$\bigcirc \leq 14$ 15-44@45-64@ $\geq 65 \quad-R_{\text {eff }}<1 \rightarrow R_{\text {eff }}>1$}

A

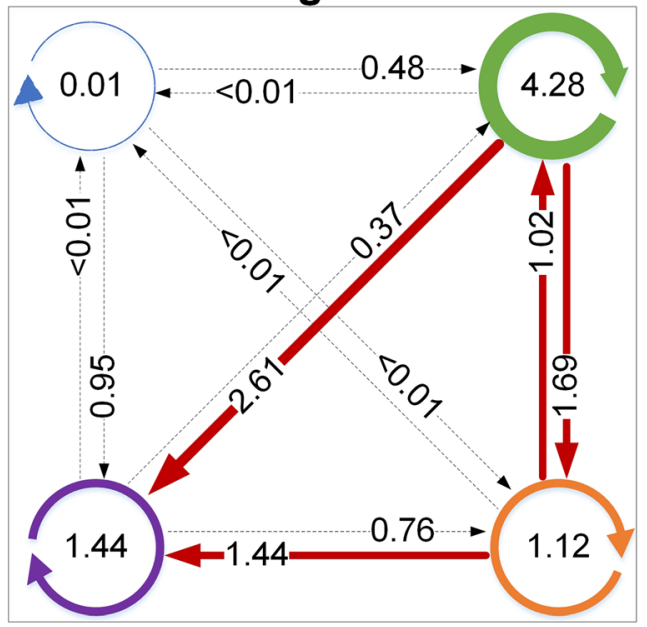

C

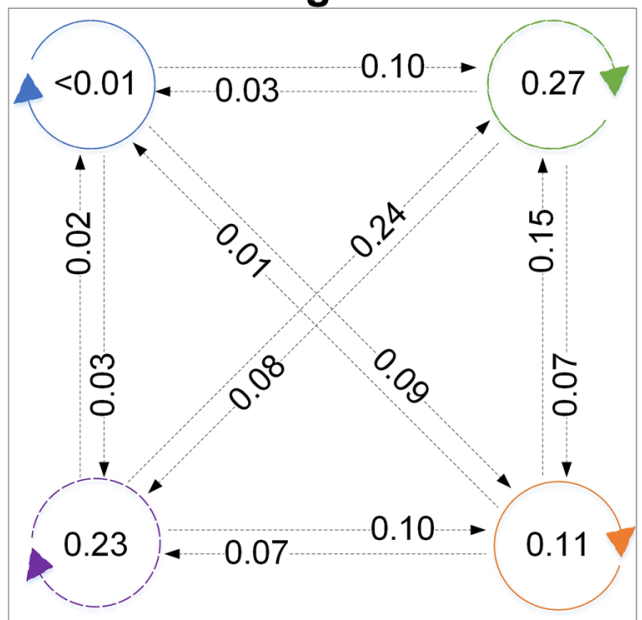

B

Stage 2

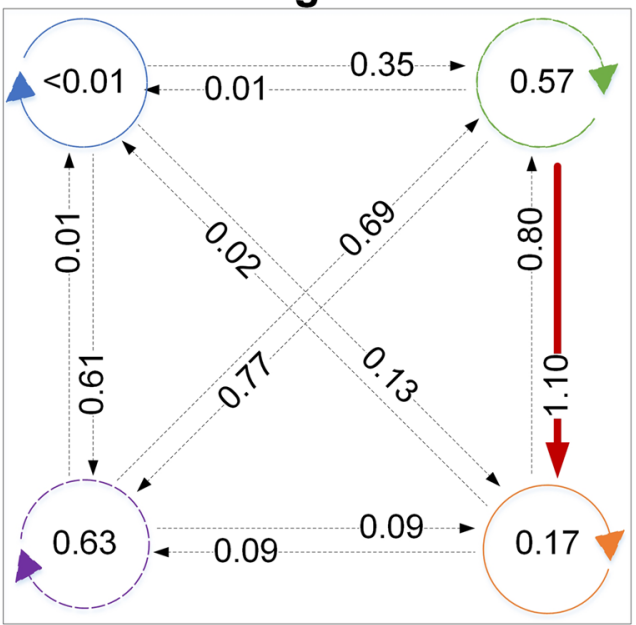

D

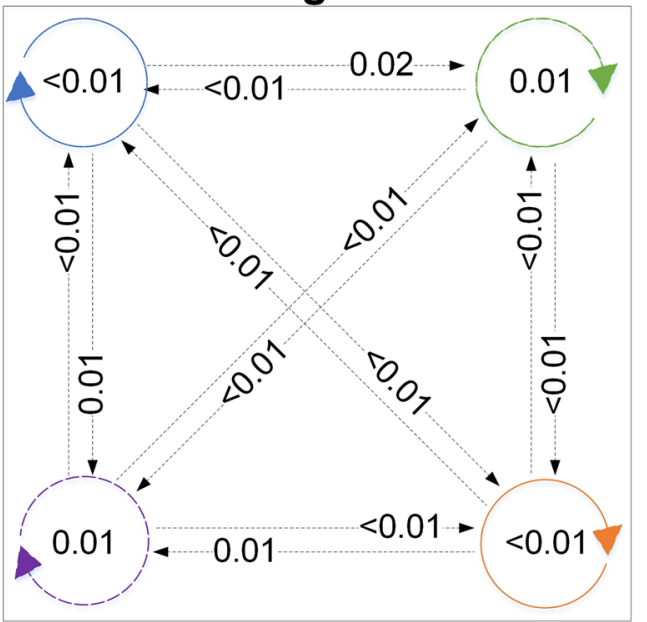

Fig. 9 The interaction of transmissibility between different age groups in four stages of Wuhan City. A Stage 1: December 2, 2019, to January 23, 2020. B Stage 2: January 24 to February 2, 2020. C Stage 3: February 3 to February 18, 2020. D Stage 4: February 19, 2020, to March 16, 2020

In scene XI of scenario I (Fig. 14), the epidemic was controlled when vaccinating $87.93 \%$ of the whole population $\left(\delta_{i}=0.05, N T=4028228, T A R=36.35 \%, D O=574\right.$, $P T=236, N P=39648)$. The optimal strategy was first to vaccinate $88.96 \%$ (Additional file 4 : Table S3) of individuals $15-44$ years old $\left(\delta_{2}=0.05, N T=4780\right.$ 964, $T A R=43.15 \%, D O=351, P T=114, N P=99288)$ and then to vaccinate $90.28 \%$ of individuals $45-64$ years old $\left(\delta_{3}=0.1, N T=6329126, T A R=57.12 \%, \quad D O=211\right.$, $P T=92, N P=274421)$. In scene XIX of scenario II (Fig. 15), the epidemic was controlled when vaccinating $96.53 \%$ of the whole population $\left(\delta_{i}=0.1, N T=4548464\right.$, $T A R=41.05 \%, D O=997, P T=162, N P=56$ 613). The optimal vaccination strategy was first to vaccinate $90.18 \%$ of individuals 15-44 years old $\left(\delta_{2}=0.1, N T=5189445\right.$, $T A R=46.83 \%, \quad D O=188, \quad P T=98, \quad N P=121 \quad 696)$ and secondly to vaccinate $88.21 \%$ of individuals aged $45-64$ years old $\left(\delta_{3}=0.1, N T=4717545, T A R=42.57 \%\right.$, $D O=217, P T=114, N P=82318)$. Our findings suggest that the vaccination priority for controlling the transmission may be vaccinating approximately $90 \%$ of individuals aged between 15 and 44 years old.

All the values of scenario I (scenes II to IX) are shown in Additional file 5: Tables S4 to S7. In scenario I of no intervention, the simulation of value was in the total population $(T N=6776$ 654, $T A R=61.16 \%, D O=205, P T=84$, 


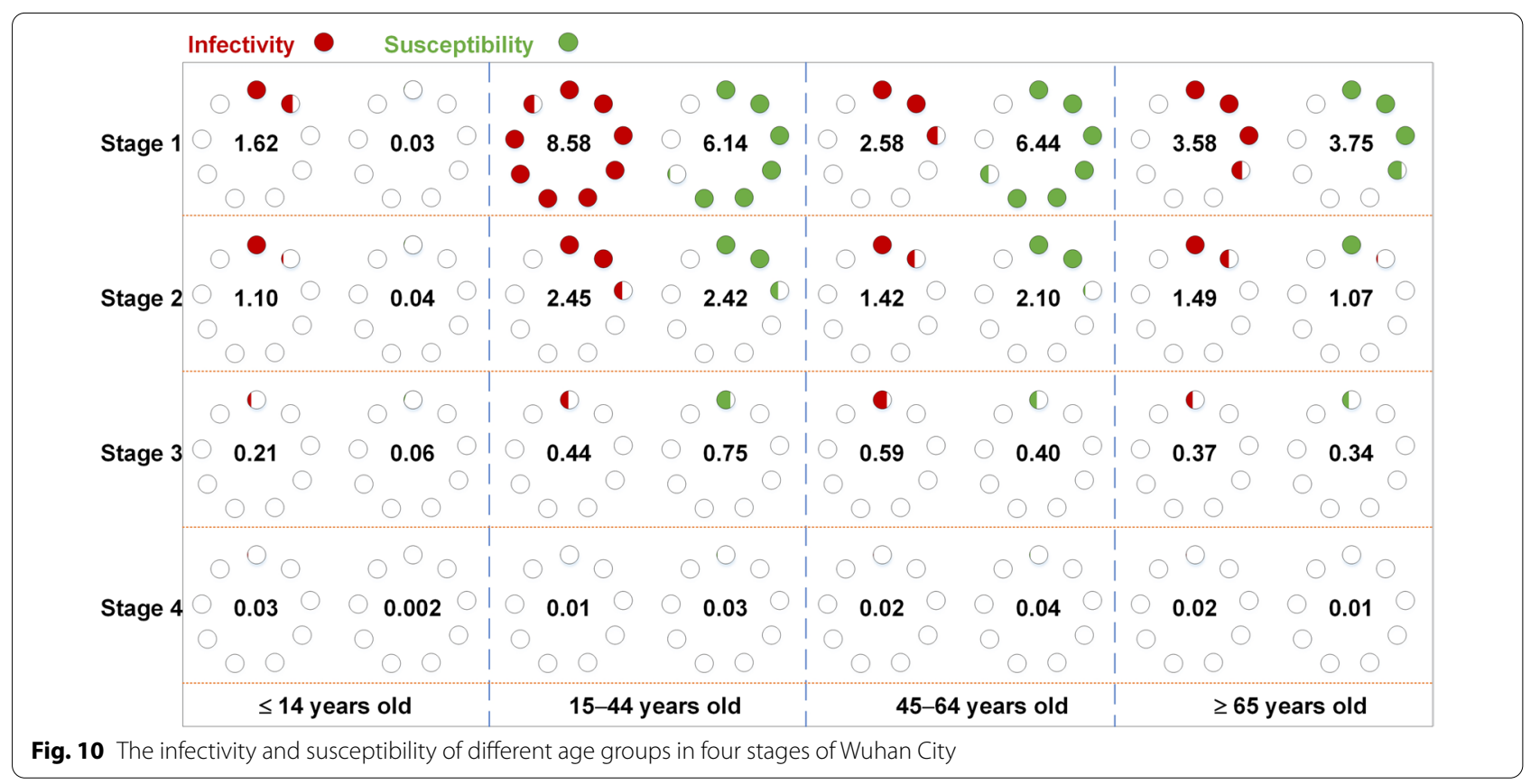

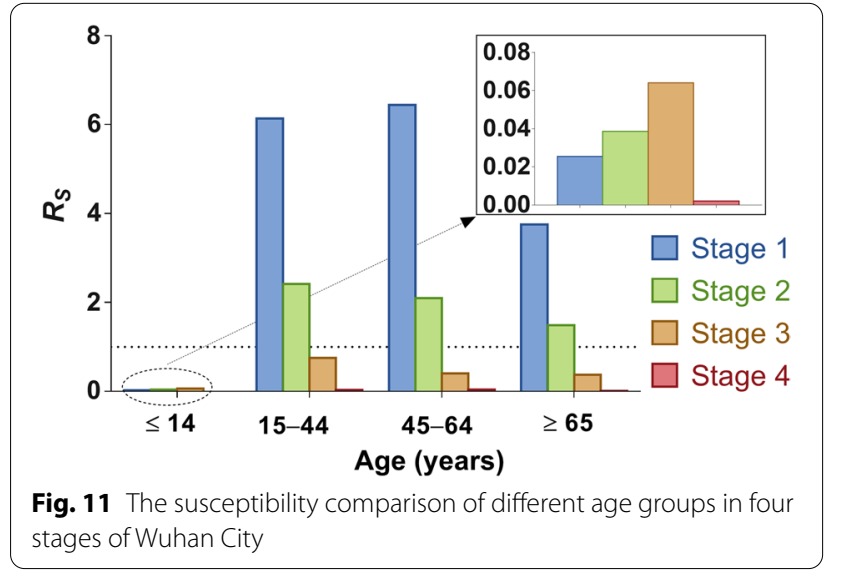

$N P=311342)$, in age group $1(T N=23595, T A R=1.88 \%$, $D O=172, P T=94, N P=792)$, group $2(T N=3136946$, $T A R=60.20 \%, D O=205, P T=87, N P=135616$ ), group $3(T N=2823084, T A R=83.66 \%, D O=108, P T=84$, $N P=148321)$ and group $4(T N=793029, T A R=64.00 \%$, $D O=138, P T=80, N P=43328)$. The simulation effects of vaccination in each age group were reduced with the increasing vaccination rate $(\delta)$, and the effects of scene II (measles vaccine) were higher than those of scenes III and IV (influenza vaccine). The best effect (Fig. 18), in which the total population was vaccinated $\left(\delta_{I}=0.1\right)$, was scene II $(T N=4722, T A R=0.04 \%, D O=177$, $P T=76, N P=68)$, followed by scene III $(T N=4026365$, $T A R=36.34 \%, D O=601, P T=278, N P=39534)$ and then scene IV $(T N=6127075, T A R=55.29 \%, D O=232$, $P T=101, N P=214987)$.

Scenes V, VI, VII, and VIII of scenario I are shown in Fig. 19, when the vaccination was simulated in each age group. The optimal strategy was to vaccinate age group 2 in the total population, especially when $\delta_{2}=0.1 \quad(T N=4802585, T A R=43.34 \%, D O=357$, $P T=119, N P=97964)$. After vaccination in age groups 1,3 , and 4 , it was less affected than the other three groups. With vaccination in age group 1 , the greatest effect $\left(\delta_{1}=0.1\right)$ was only for itself $(T N=6942$, $T A R=0.55 \%, D O=161, P T=97, N P=229)$. With vaccination in age group 2 , the greatest effect $\left(\delta_{2}=0.1\right)$ was only for itself (TN=2 $310030, T A R=44.33 \%$, $D O=357, P T=131, N P=42553)$. With vaccination in age group 3 , the greatest effect $\left(\delta_{3}=0.1\right)$ was only for itself $(T N=2419225, T A R=71.69 \%, D O=113$, $P T=91, N P=120$ 356). With vaccination in age group 4 , the greatest effect $\left(\delta_{4}=0.1\right)$ was only for itself $(T N=778$ 980, $T A R=62.86 \%, \quad D O=188, \quad P T=89$, $N P=34451)$.

The simulation of different VE and the relative immune rates in the total population is shown in Fig. 20. The best simulation effect of VE was $\lambda=1$ and $\lambda=0.9(T N=0, \quad T A R=0, \quad D O=0, \quad P T=0, \quad N P=0)$, and followed by $\lambda=0.8(T N=531227, T A R=4.79 \%$, $D O=3804, P T=1862, N P=455)$. The best simulation effect of the relative immune rate was $\phi=1$ $(T N=4026318, T A R=36.34 \%, D O=645, P T=322$, $N P=39533)$ and the worst effect of the relative 


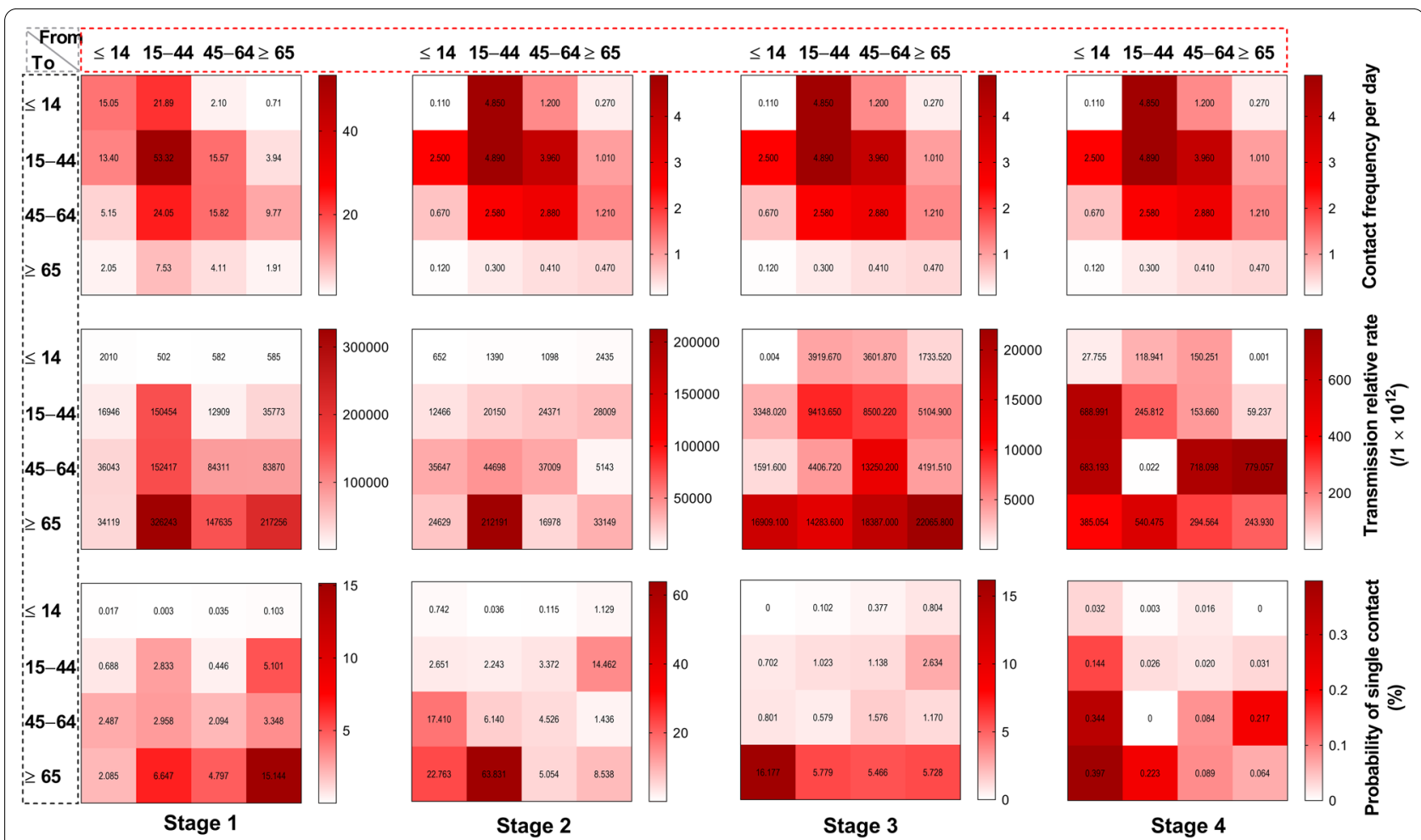

Fig. 12 The risk of infected probability in different age groups of four stages

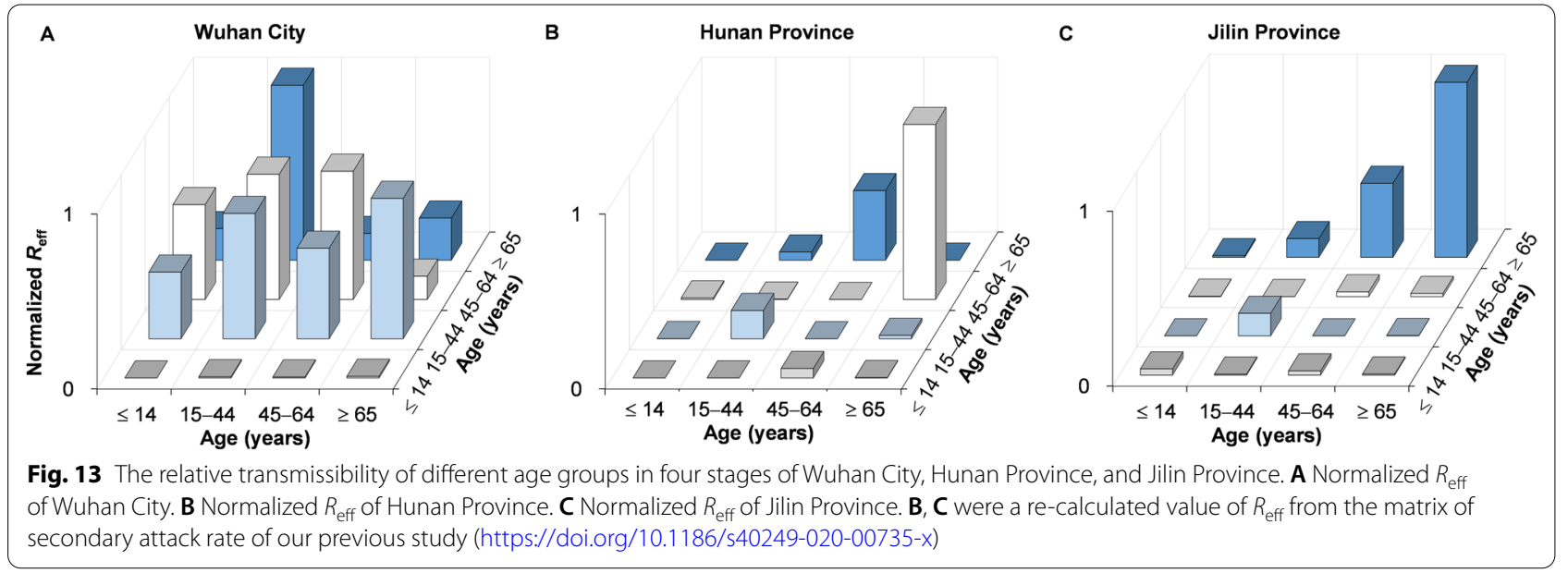

immune relative was $\phi=0.033 \quad(T N=4 \quad 078 \quad 737$, $T A R=36.81 \%, D O=494, P T=179, N P=43113)$.

All the values of scenario II (scenes XI to XV) are shown in Additional file 6: Tables S8 to S11. Vaccination of the total population in scenario II (Fig. 21), the total number of cases was reduced by the rising vaccination rates. The best simulation effect was when $\delta_{i}=0.1$ for the number of cases in total population
$(T N=4548$ 464, $T A R=41.05 \%, D O=997, P T=162$, $N P=56613)$, respectively.

Regarding the vaccination of each age group in scenario II (Fig. 22), the optimal strategy was vaccination of age group 2 for the total population, especially when $\delta_{2}=0.1 \quad(T N=5189 \quad 459, \quad T A R=46.83 \%, D O=190$, $P T=97, N P=121696)$ and age group 3, especially when $\delta_{3}=0.1$ for the total population $(T N=4624965$, 




$T A R=41.74 \%, \quad D O=219, \quad P T=97, \quad N P=378 \quad 275)$. Less effects were found with vaccinated age group 1 $(T N=6429821, T A R=58.03 \%, D O=1254, P T=62$, $N P=400251)$ and $4(T N=6499928, T A R=58.66 \%$, $D O=1258, P T=64, N P=403$ 077).

\section{Effectiveness of vaccination on the disease severity}

The simulated total number of deaths decreased with increasing age and vaccination rate (Fig. 23). The simulated total number of deaths was 209984 in the total population, and it was 19918 300, 73800 and 117686 in age groups 1, 2, 3, and 4, respectively. The best simulation for the total population was obtained with the vaccination of age group $4(\delta=0.1$, $N D=133230)$, followed by vaccinating age group 3 $(\delta=0.1, N D=161586)$. Moreover, the best simulation for decreasing the death rate of age group 1 was vaccination of group $1(\delta=0.03, N D=0)$, when the members of age group 2 were vaccinated $(\delta=0.1, N D=4151)$, when the members of age group 3 were vaccinated $(\delta=0.1, N D=25385)$, and when the members of age group 4 were vaccinated $(\delta=0.1, N D=40933)$. Our findings suggest that vaccination protocols should prioritize older populations.

\section{Discussion}

When modeling the transmissibility, although the transmission pattern varied from one area to another, the highest transmissibility was found in individuals aged 15-44 years, while the highest risk of infection was among the elderly population. Therefore, the optimal vaccination strategy for controlling the transmission of COVID-19 should be to first vaccinate about $90 \%$ of 15-44 years old, while for reducing the disease severity, the vaccination priority should be on the older population.

Similar to our previous study, the age-specific SEIAR model fits the data well $[11,12]$. There is no doubt that it has a great impact on controlling the epidemics in Wuhan City [2], while accounting for key intervention measures such as travel ban, case isolation, and increasing social distancing, among others.

Before the travel ban of Wuhan City, the highest transmissibility was found within the most socially 


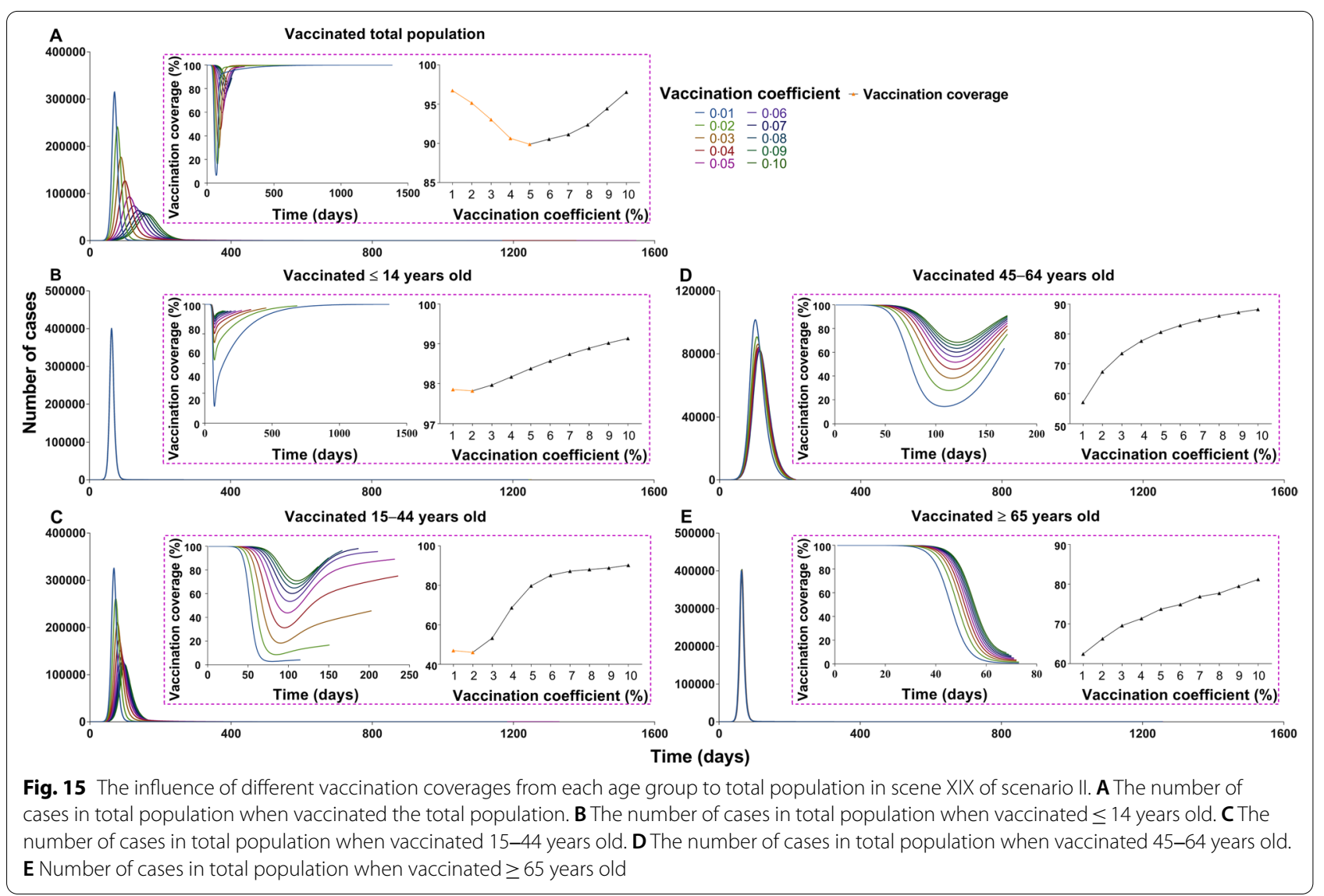

active age group, those aged 15-44 years old. The highest contact frequency in this group was directly linked to its high level of contact with other age groups [51]. January 25, 2020, corresponding to the Spring Festival (Chinese Lunar New Year celebration), an event where there would be a large number of travelers, such as workers and students returning home, present within the first 15 days of the festival $[56,57]$. In China, the secondary cases were mostly caused by travelers, especially middle-aged and elderly people [31, 58]. Meanwhile, our study indicated that the susceptibility of those 15-65 years of age was higher than those in other age groups. If we set the period before the travel ban in Wuhan City as a baseline, the highest level of interaction was among those 15-65 years of age. Following the implementation of control measures during the travel ban period, transmissibility decreased in Wuhan City. The $R_{\mathrm{S}}$ value for the population below 14 years of age was 0.0255 , and it gradually increased in stages 2 $\left(R_{\mathrm{S}}=0.0386\right)$ and $3\left(R_{\mathrm{S}}=0.0641\right)$. This finding suggests that the risk of transmission would be amplified after intervention for those below 14 years of age, especially during the travel ban and home quarantine. A previous study indicated that children and adults face a similar risk of infection [58]. Meanwhile, some surveys also reported that the transmission of SARS-CoV-2 largely occurred within the family $[60,61]$. Therefore, it is important to prevent transmission within families after home isolation.

Although the contact frequency among $\geq 65$ years old is very low $(\alpha=1.91)$, this age class displayed the highest probability of infection upon single contact, that is, it is the most susceptible age class, $(q=15.144 \%)$ before the travel ban. In China, there are many cluster activities among the elderly, such as square dancing, dinner parties, and card games. These cluster activities have promoted long-term and effective exposure between people, thereby increasing the risk of infection [61]. Meanwhile, in the other three stages, the elderly are also at a very high risk of being infected by other age groups. This suggests that social distancing should be increased and cluster activities should be decreased to control COVID-19, especially in the elderly.

However, because of the varied results with influenza incidence in various age groups $[62,63]$, the transmission interaction in the age group was not a constant pattern. There is a major difference in age-specific transmission between Wuhan City, Hunan Province, and Jilin 


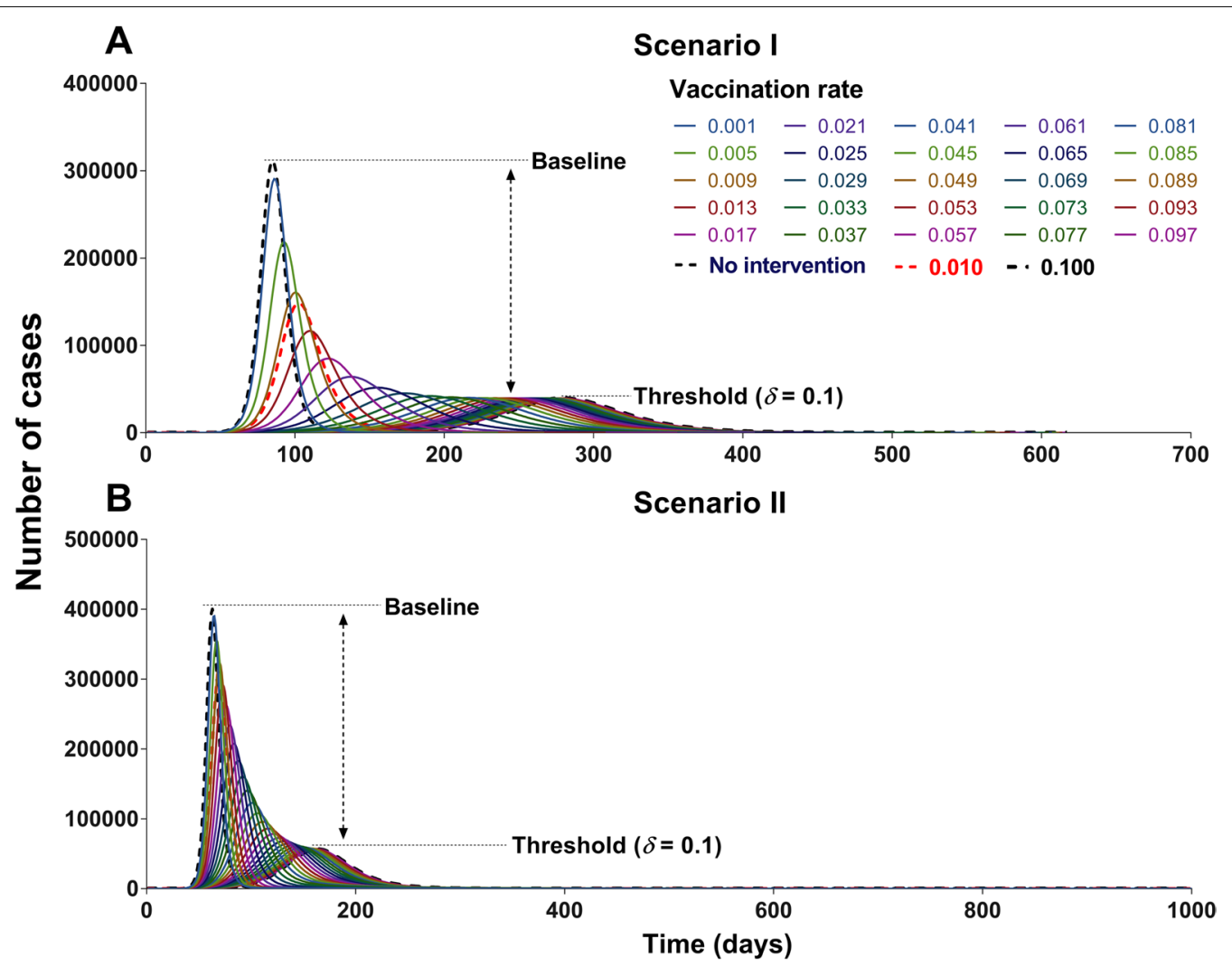

Fig. 16 Simulated the effects of different vaccination rates in scene I of scenario I and scene XI scenario II. A Different vaccination rates of scenarios I. B Different vaccination rates of scenarios II
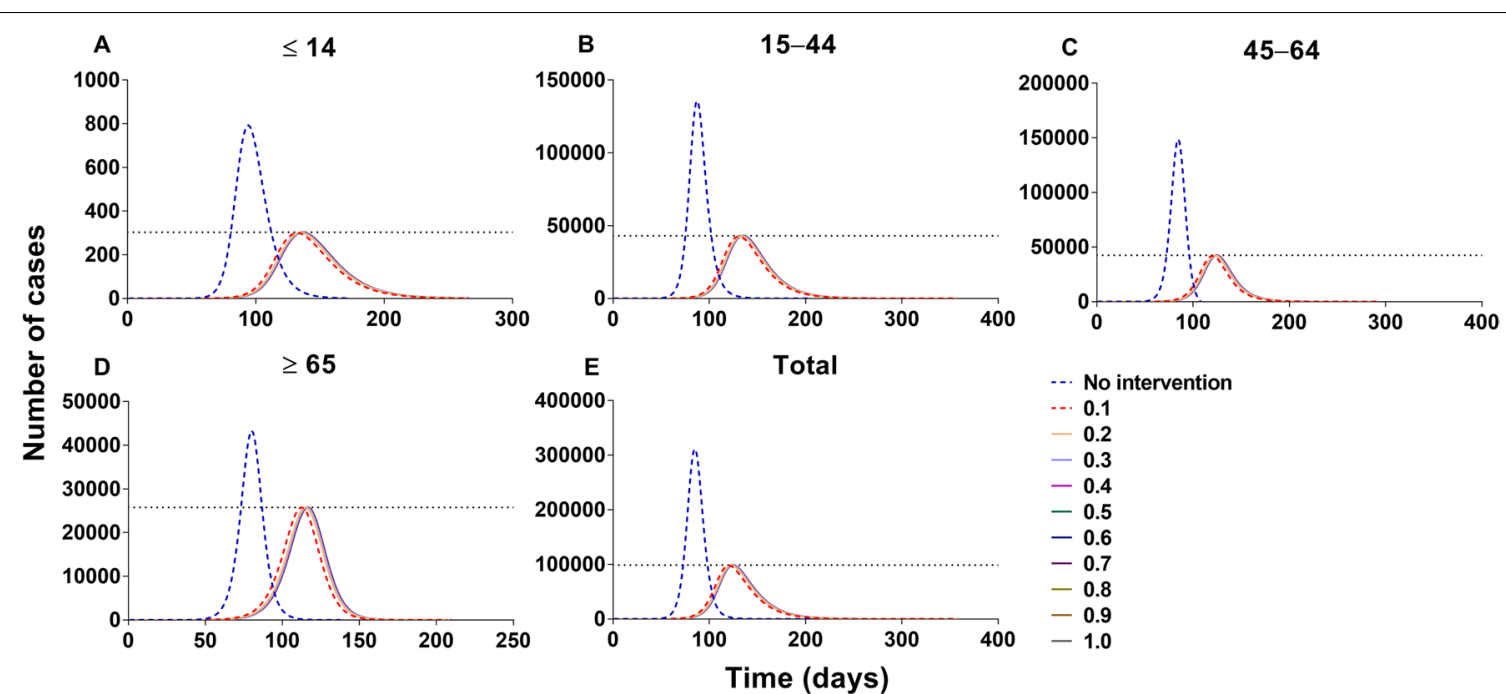

No intervention

0.1

$-0.2$

$-0.3$

$-0.4$

$-0.5$

$-0.6$

$-0.7$

$-0.8$

$-0.9$

Time (days)

Fig. 17 Simulated the different vaccination rates in each age group. A Vaccinated individuals $\leq 14$ years old. B Vaccinated individuals $15-44$ years old. C Vaccinated individuals 45-64 years old. D Vaccinated individuals $\geq 65$ years old. E Vaccinated total population 


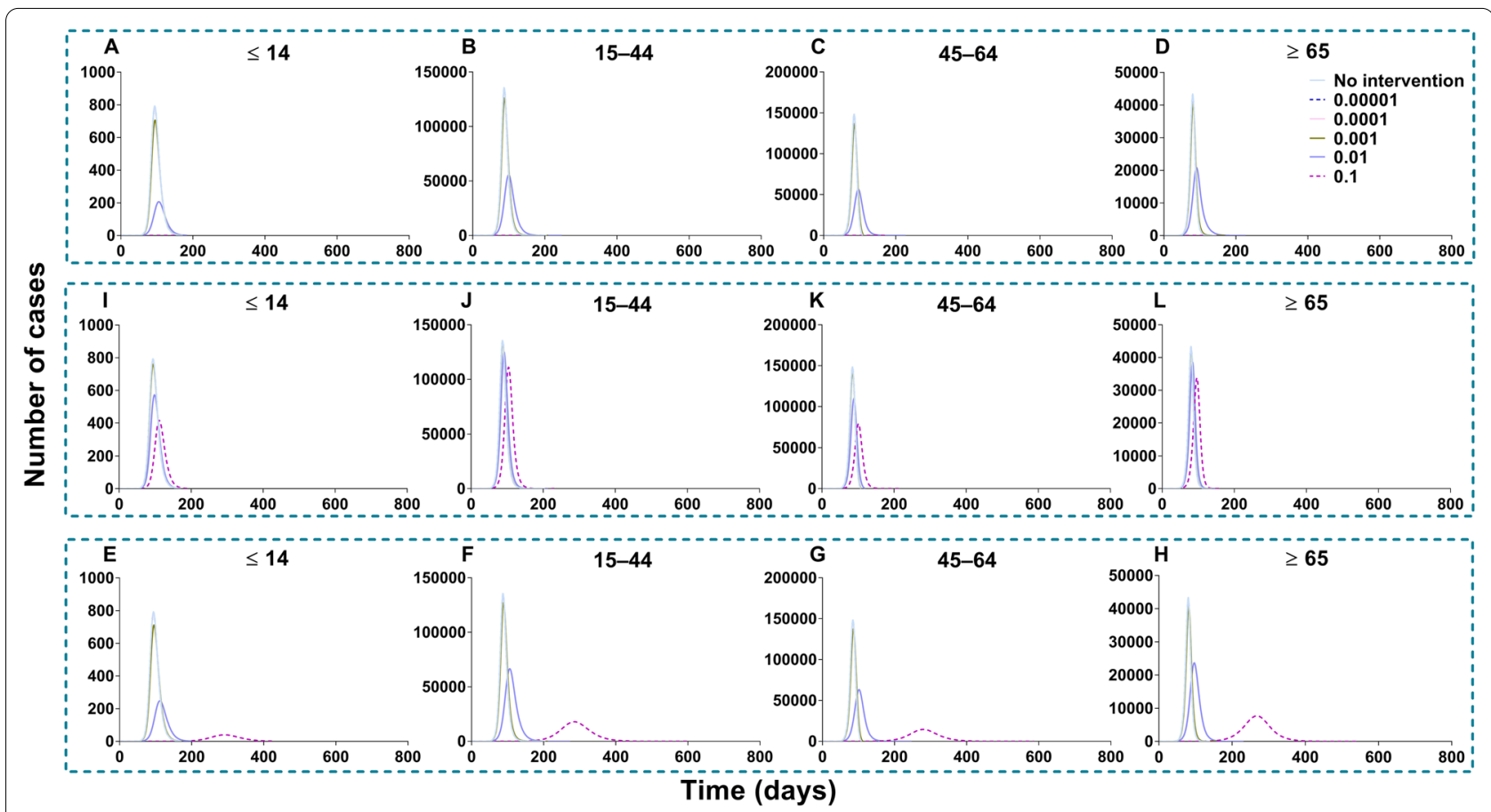

Fig. 18 Simulated the vaccine effects same as measles and influenza vaccine in scene II, III, and IV of scenario I. A Measles vaccine vaccinated total population $(\mathrm{VE}=90 \%$ ). B High VE of influenza vaccine vaccinated total population (VE $=70 \%)$. C Low VE of influenza vaccine vaccinated total population $(\mathrm{VE}=30 \%)$

Province. This difference might be related to differing societal factors such as economy, culture, and demography. The population density and economic status of Wuhan City was higher than those of Hunan Province and Jilin Province. Some studies have indicated that there are differences in COVID-19 outbreaks in every province of China [58]. In addition, the epidemics of Hunan Province and Jilin Province were local infections mostly caused by imported cases. Therefore, age-specific transmission patterns need to be further explored and compared between different areas.

Currently, the mRNA vaccine of BioNTech and Moderna reported great effect (VE $>90 \%$ ) for controlling the ancestral virus [5-7]. However, the effectiveness of the vaccine has been gradually decreasing with the mutation of the virus, exemplified by just $75 \%$ (95\% CI: $68 \%-79 \%$ ) of ChAdOx1 $\mathrm{nCoV}-19$ for the Delta variant [8]. In this study, we considered VE-like vaccine efficacy of measles $(\mathrm{VE}=90 \%)$, influenza $\mathrm{B}(\mathrm{VE}=70 \%)$ and influenza $\mathrm{A}$ $(\mathrm{VE}=30 \%)$. We obtained similar age-optimized vaccination results for the above three conditions. Our findings suggest that the optimal vaccination proposal is consistent across age groups regardless of vaccine efficacy. VE is at least $70 \%$ and $90 \%$, respectively, which is consistent with the actual vaccine effects. Therefore, the evaluation of COVID-19 vaccines could be referred to as the vaccine against influenza and measles. This study explored two scenarios including 19 scenes to explore the unknown future vaccine efficacy and behavior and the optimal strategy to control COVID-19. The simple model in the scenario I aim to cover a wide range of VE, and the more complex model in scenario II aims to explore the decreasing relative infectivity and susceptibility. Indeed, the vaccine for measles has a good efficacy [44], but this might not be the case for COVID-19 vaccines. Therefore, when simulating the efficacy of COVID-19 as that of the seasonal influenza vaccine, the vaccination coverage should be at least $87.93 \%$ of the total population in scene XI of scenario I and $96.53 \%$ of the total population in scene XIX of scenario II. Conversely, a specific study indicated that a vaccine with an efficacy $\geq 50 \%$ would be enough to mitigate the pandemic and the vaccine should be allocated for use in the elderly first $[13,64]$.

To increase the accuracy of the simulation, we further added asymptomatic infection as a factor and simulated the vaccination outcomes after estimation of transmissibility in different age groups according to the real-life situation.

Considering that it is difficult to vaccinate nearly $90 \%$ of the total population, we simulated the vaccination rate in each age group. Although several studies, including ours, indicated a high risk of infection in the 


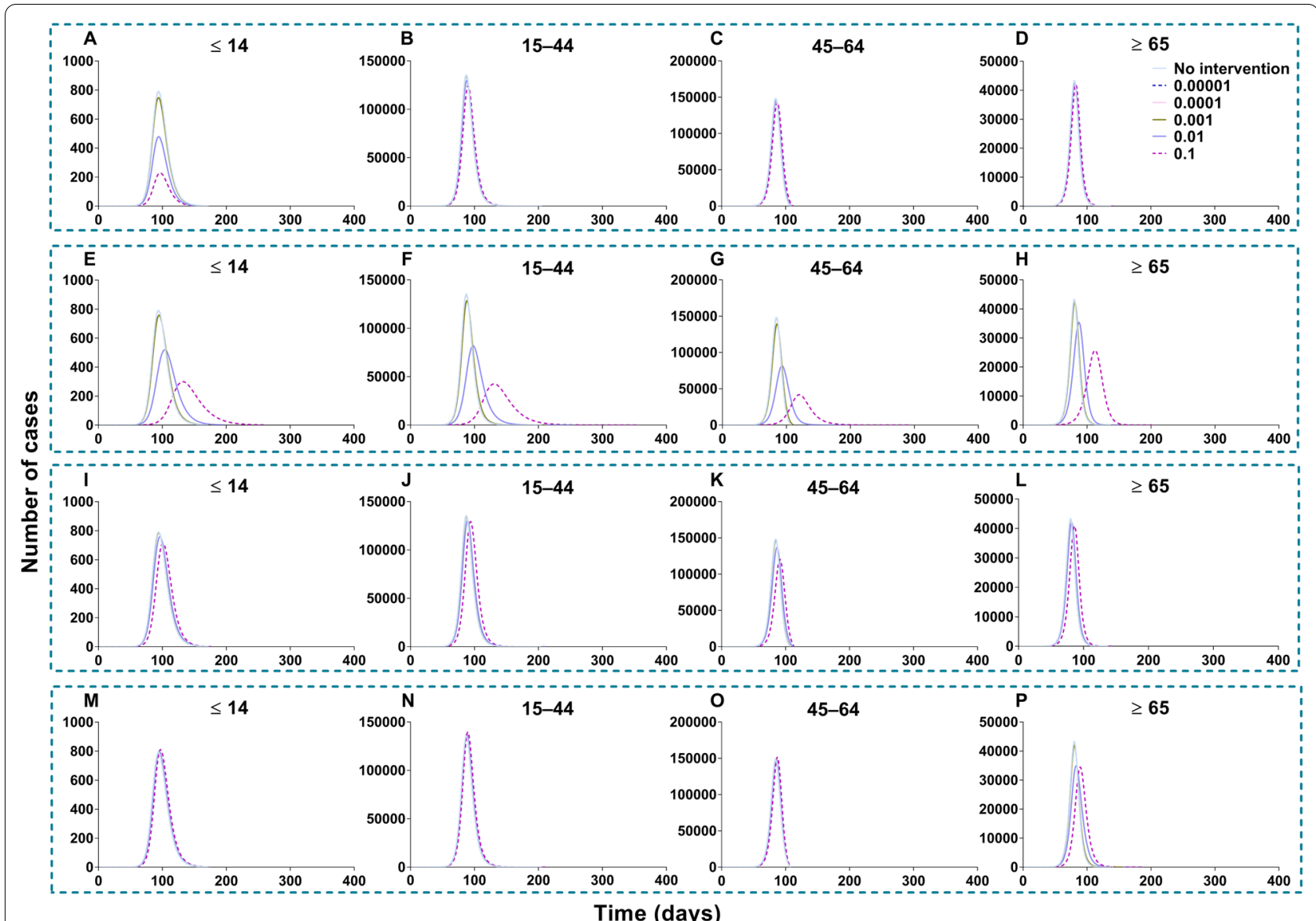

Fig. 19 Simulated the effects vaccinated each age group in scene V, VI, VII, and VIII of scenario I. A-D Vaccinated individuals $\leq 14$ years old. E-H Vaccinated individuals 15-44 years old. I-L Vaccinated individuals 45-64 years old. M-P Vaccinated individuals $\geq 65$ years old

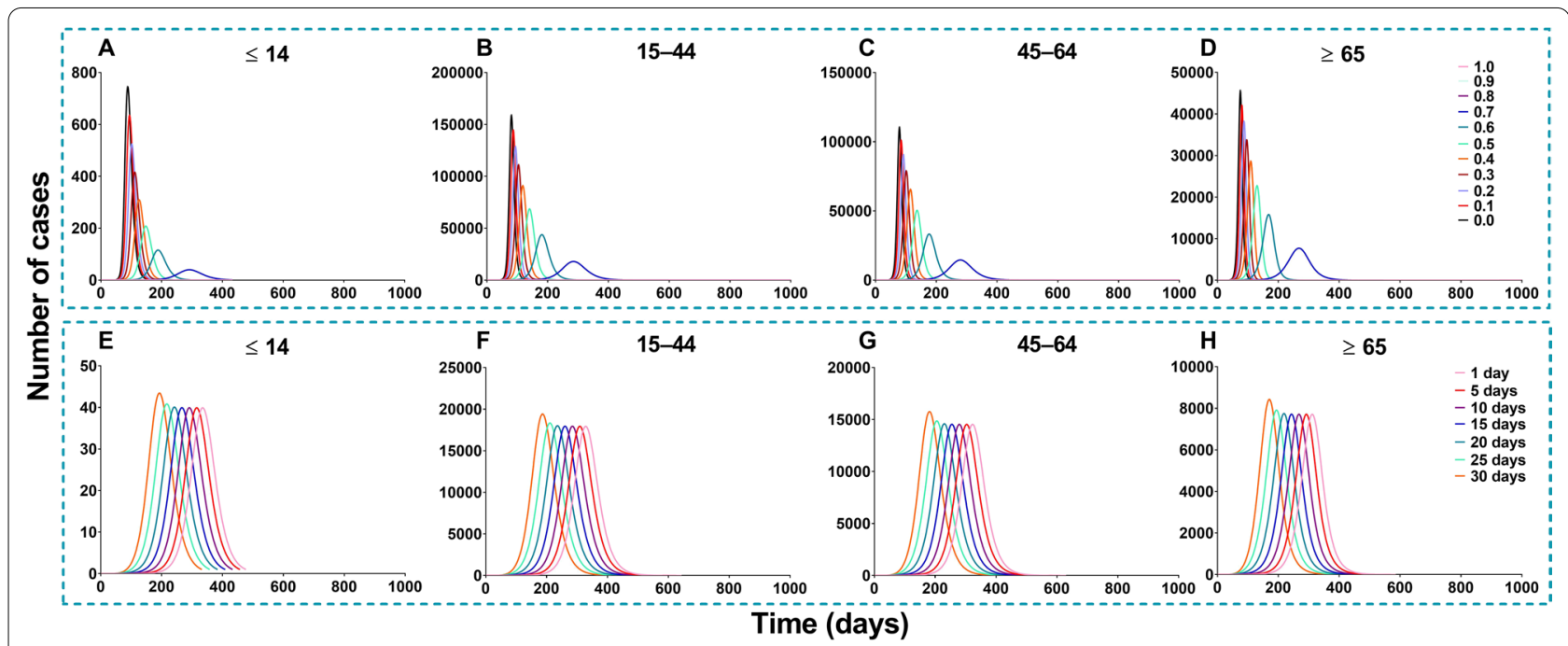

Fig. 20 Simulated the different VE and immune relative rates in scenes IX and X of scenario I. A-D Different VE vaccinated total population. E-H With immunity after different periods vaccinated total population 


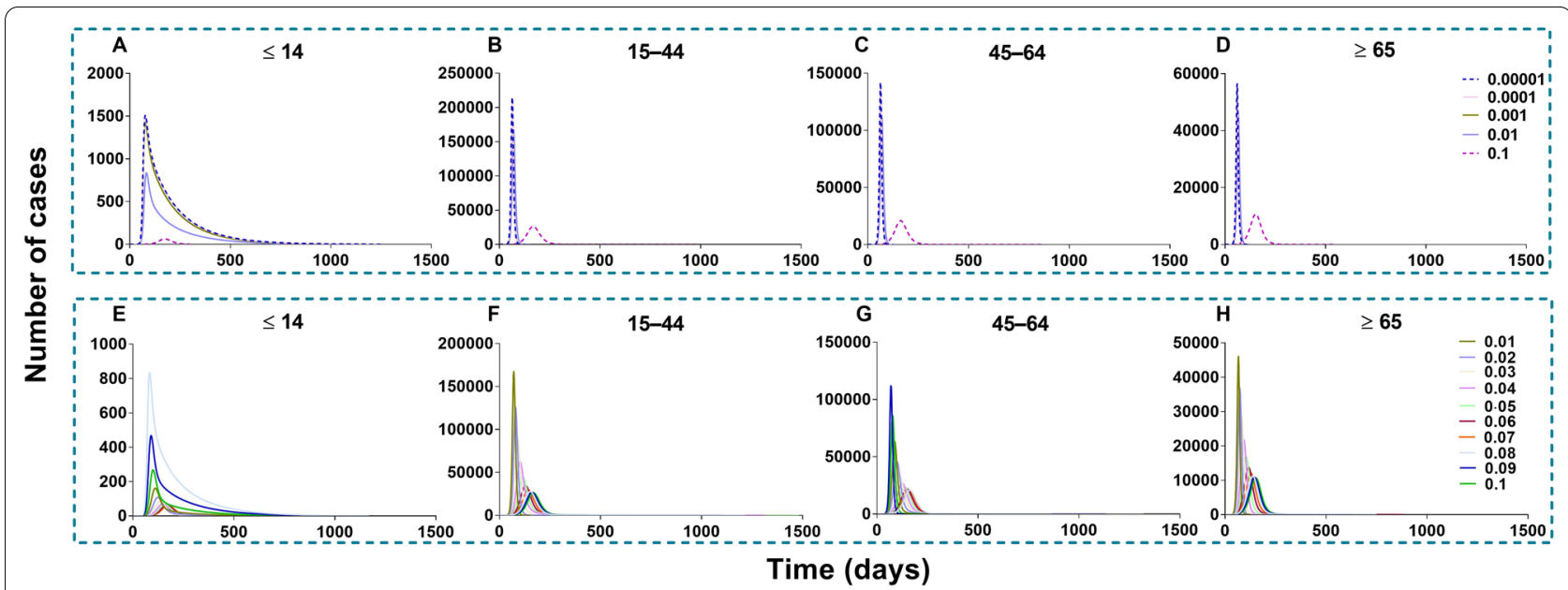

Fig. 21 Simulated the different vaccination rates when vaccinated total population in scene XII of scenario II. A-D Vaccination rate was 0.0001 , $0.001,0.01$ and 0.1 , respectively. The number of cases in the no-vaccinated group when vaccinated total population. $\mathbf{E}-\mathbf{H}$ Vaccination rate from 0.01 to 0.1

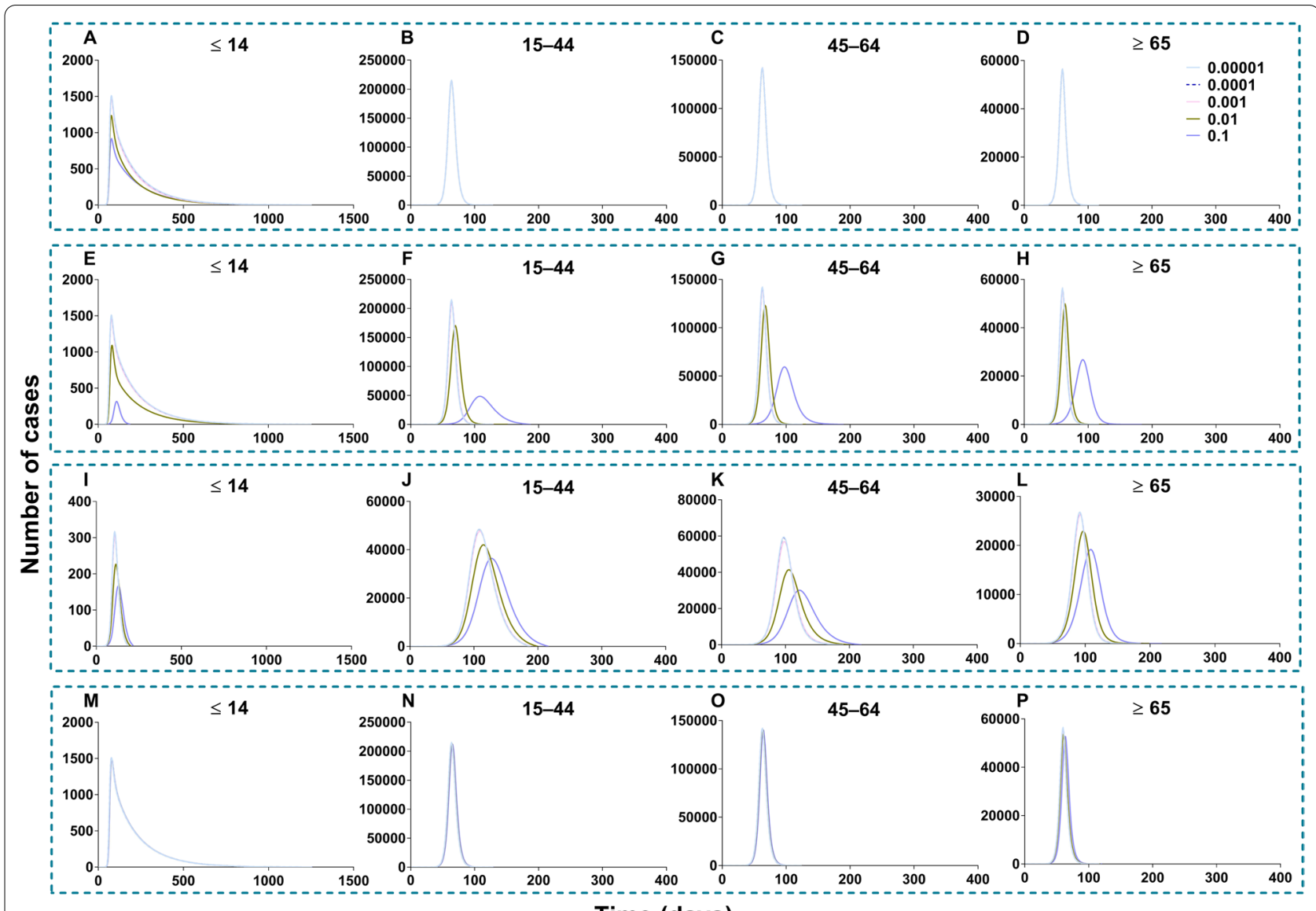

Time (days)

Fig. 22 Simulated the different vaccination rates in each age group in scenes XI, XII, XIII, and XIV of scenario II. A-D Vaccinated individuals $\leq 14$ years old. E-H Vaccinated individuals 15-44 years old. I-L Vaccinated individuals 45-64 years old. M-P Vaccinated individuals $\geq 65$ years old 


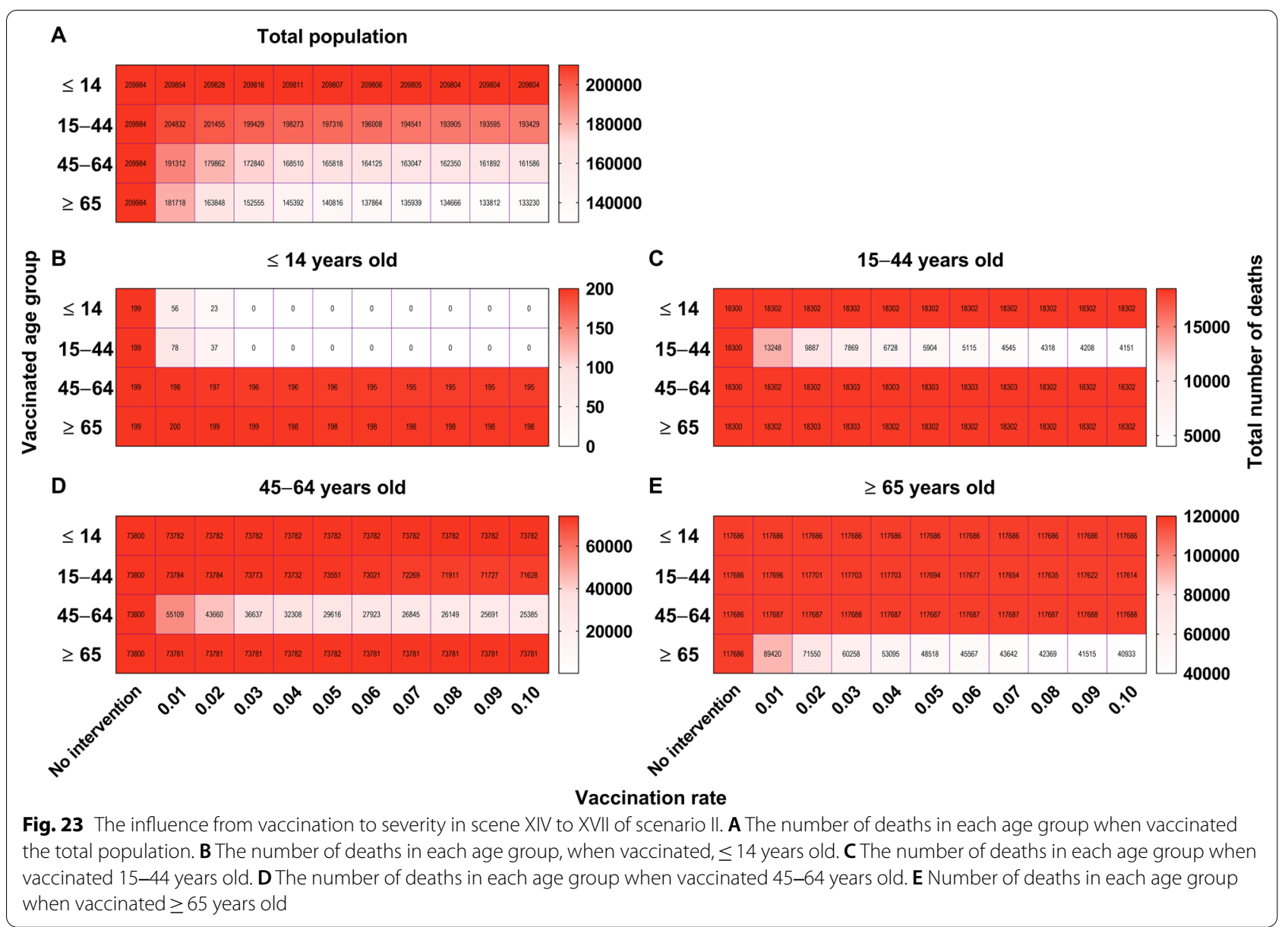

elderly [12, 52], our results of vaccination modeling in each age group showed that the optimal strategy was to first vaccinate individuals aged $15-44$ years. Whether this simulation corresponds to scenario I or scenario II, it can significantly reduce the number of cases in the overall population. The main reason is the very high transmissibility (including susceptibility and infectivity) of SARS-CoV-2 in individuals of the 15-44 age group. Furthermore, the age class with the highest contact frequency were those 15-44 years old [52]. The higher-risk occupations included car, taxi, and van drivers, shop sales associates, domestic housekeepers, religious professionals, etc. [65], who belonged mainly in the 15-44-year-old group. Therefore, a very positive outcome in terms of transmission can be expected when vaccinating first this age group, especially high-risk workers such as healthcare workers, drivers, transport workers, and services and sales workers. If the objective is to reduce mortality, the strategy should be to first target the age group above 65 [13, 29, 66]. In contrast, our results showed that we should not limit the vaccination to a given class but instead, to optimize the efficacy, vaccinate in a specific order: first those $\geq 65$ years old, followed by those 45-64 years old, then those 15-44 years old, and finally those $\leq 14$ years old.

However, transmission patterns differ from one area to another $[67,68]$. We highlight this heterogeneity in transmission, especially in terms of age interaction, because these results may be optimal for Wuhan City but perhaps less suitable for other regions. Therefore, we should estimate a strategy of vaccination optimization after having sufficiently clarified age-specific transmission interactions in different areas. In particular, we should estimate the transmissibility and simulate vaccination outcomes in different age groups in different regions. We limited the real-time vaccinating process simulated in our model, $\delta=0.1$ means nearly $10 \%$ of the total population is vaccinated per day. It is necessary to simulate an initial proportion of the immune population. A study reported the impact of policy interventions (like home quarantine) and meteorological factors (such as air index, temperature, precipitation, and relative humidity) on vaccination effectiveness [69]. Although an immune barrier has been established in a proportion of people, we need to 
consider the impact of meteorological factors on transmission and vaccination and simulate the combined effect between vaccination and non-pharmacological interventions in future work.

\section{Limitations}

In the study, we should collect most COVID-19 data to compare the different age-specific transmission in various areas. Furthermore, we are limited to not analyzing the impact of meteorological factors on transmission and vaccination. At last, we should re-evaluate the optimization strategy based on the current immune barrier.

\section{Conclusions}

The highest transmissibility was observed in those aged 15-44 years and the risk of infection probability was highest in the elderly. In China, approximately $85 \%$ of the total population should be vaccinated to effectively build an immune barrier and take reopening under consideration. The optimized strategy to control transmission was to first vaccinate about $90 \%$ of individuals aged 15-44 years, but for reducing the disease severity, the elderly should be vaccinated first.

\footnotetext{
Abbreviations

COVID-19: Coronavirus disease 2019; NPIs: Non-pharmaceutical interventions; VE: Vaccine efficacy; SARS-CoV-2: Severe acute respiratory syndrome coronavirus 2; SEIR: Susceptible-exposed-infectious-removed; SEIAR:

Susceptible-exposed-symptomatic-asymptomatic-recovered/removed; $R_{\text {eff: }}$ : Effective reproduction number; $R_{i}$ : Infectivity; $R_{5}$ : Susceptibility; q: Probability of infection from a single contact; NT: Total number of new cases; TAR: Total attack rate; NP: Number of new cases at peak; DO: Duration of outbreak; PT: Peak time; ND: Total number of deaths; $R^{2}$ : Coefficient of determination.
}

\section{Supplementary Information}

The online version contains supplementary material available at https://doi. org/10.1186/s40249-021-00922-4.

Additional file 1: Table S1. The data of illness onset of four age groups in Wuhan City.

Additional file 2: Text S1. Equations of Model 2 and Model 3.

Additional file 3: Table S2. Description and source of parameters in the age-specific model.

Additional file 4: Table S3. Five indicators and vaccination coverage of scene XI and scene XIX.

Additional file 5: Tables S4-S7. Simulation different vaccination scenes. Additional file 6: Tables S8-S11. Simulated different vaccination rates.

Acknowledgements

Not applicable.

\section{Authors' contributions}

TMC, RF, and WLH designed research; YN and TMC collected data; TMC, YN, LL, WLH, and TLY conceived the experiments, QQH, MJC, QPC, LZ, JR, CLS, SNL, $Y W$, JWX, YZZ, XCL, MY, JFH, WKL, BD, CL, ZYL, PHL, YHS, and BHZ conducted the experiments and analyzed the results; TMC, ZYZ, RF and LL wrote the manuscript. All authors read and approved the final manuscript.

\section{Funding}

This study was partly supported by the Bill \& Melinda Gates Foundation (Grant INV-005834 to T.C.), the Science and Technology Program of Fujian Province (Grant 2020Y0002 to T.C.), and NHC Key Laboratory of Echinococcosis Prevention and Control (Grant 2020WZK2001 to T.C.). The funders had no role in study design, data collection and analysis, decision to publish, or preparation of the manuscript.

\section{Availability of data and materials}

All relevant data are within the paper and its Additional Information.

\section{Declarations}

Ethics approval and consent to participate

The study was approved by the Ethics Committee of School of Medicine, Xiamen University.

\section{Consent for publication}

Not applicable.

\section{Competing interests}

The authors declare that they have no competing interests.

\section{Author details}

${ }^{1}$ State Key Laboratory of Molecular Vaccinology and Molecular Diagnostics, School of Public Health, Xiamen University, 4221-117 South Xiang'an Road, Xiang'an District, Xiamen 361102, Fujian, People's Republic of China. ${ }^{2}$ Cirad, UMR 17, Intertryp, Université de Montpellier, Montpellier, France. ${ }^{3}$ Chinese Center for Disease Control and Prevention, Beijing, People's Republic of China. ${ }^{4}$ Division of Public Health, School of Medicine, University of Utah, Utah, USA. ${ }^{5}$ Medical Insurance Office, Xiang'an Hospital of Xiamen University, Xiamen, Fujian, People's Republic of China. ${ }^{6}$ Department of Data Science, College of Natural Sciences, Colorado State University, Fort Collins, CO, USA. ${ }^{7}$ Fujian Provincial Center for Disease Control and Prevention, 76 Jintai Road, Gulou District, Fuzhou, Fujian, People's Republic of China.

Received: 11 August 2021 Accepted: 15 December 2021

Published online: 28 December 2021

\section{References}

1. Organization WH. WHO coronavirus disease (COVID-19) dashboard. https://covid19.who.int/2020. Accessed 16 Nov 2021.

2. Pan A, Liu L, Wang C, Guo H, Hao X, Wang Q, et al. Association of Public Health Interventions With the Epidemiology of the COVID-19 Outbreak in Wuhan. China JAMA. 2020;323:1915-23.

3. Control ECfDPa. Guidelines for non-pharmaceutical interventions to reduce the impact of COVID-19 in the EU/EEA and the UK. ECDC: Stockholm. 2020.

4. Song H. COVID-19 vaccine: China's public good versus 'America First'. https://www.globaltimes.cn/content/1198456.shtml2020. Accessed 16 July 2021.

5. Tenforde MW, Olson SM, SelfWH, Talbot HK, Lindsell CJ, Steingrub JS, et al Effectiveness of Pfizer-BioNTech and Moderna Vaccines Against COVID-19 Among Hospitalized Adults Aged $>/=65$ Years - United States, JanuaryMarch 2021. MMWR Morb Mortal Wkly Rep. 2021;70:674-9.

6. Thompson MG, Burgess JL, Naleway AL, Tyner HL, Yoon SK, Meece J, et al. Interim Estimates of Vaccine Effectiveness of BNT162b2 and mRNA-1273 COVID-19 Vaccines in Preventing SARS-CoV-2 Infection Among Health Care Personnel, First Responders, and Other Essential and Frontline 
Workers - Eight U.S. Locations, December 2020-March 2021. MMWR Morb Mortal Wkly Rep. 2021;70:495-500.

7. Thompson MG, Burgess JL, Naleway AL, Tyner H, Yoon SK, Meece J, et al. Prevention and Attenuation of Covid-19 with the BNT162b2 and mRNA1273 Vaccines. N Engl J Med. 2021;385:320-9.

8. Lopez Bernal J, Andrews N, Gower C, Gallagher E, Simmons R, Thelwall S, et al. Effectiveness of Covid-19 Vaccines against the B16172 (Delta) Variant. N Engl J Med. 2021;385:585-94.

9. Dagan N, Barda N, Kepten E, Miron O, Perchik S, Katz MA, et al. BNT162b2 mRNA Covid-19 Vaccine in a Nationwide Mass Vaccination Setting. N Engl J Med. 2021;384:1412-23.

10. Kucharski AJ, Russell TW, Diamond C, Liu Y, Edmunds J, Funk S, et al. Early dynamics of transmission and control of COVID-19: a mathematical modelling study. Lancet Infect Dis. 2020;20:553-8.

11. Chen TM, Rui J, Wang QP, Zhao ZY, Cui JA, Yin L. A mathematical model for simulating the phase-based transmissibility of a novel coronavirus. Infect Dis Poverty. 2020;9:24.

12. Zhao ZY, Zhu YZ, Xu JW, Hu SX, Hu QQ, Lei Z, et al. A five-compartment model of age-specific transmissibility of SARS-CoV-2. Infect Dis Poverty. 2020;9:117.

13. Matrajt L, Eaton J, Leung T, Brown ER. Vaccine optimization for COVID-19, who to vaccinate first? MedRxiv. 2020. https://doi.org/10.1101/2020.08. 14.20175257.

14. Arolas HPI, Acosta E, Myrskyla M. Optimal vaccination age varies across countries. Proc Natl Acad Sci U S A. 2021;118:98.

15. Moore S, Hill EM, Dyson L, Tildesley MJ, Keeling MJ. Modelling optimal vaccination strategy for SARS-CoV-2 in the UK. PLoS Comput Biol. 2021;17:e1008849.

16. Shim E. Optimal Allocation of the Limited COVID-19 Vaccine Supply in South Korea. J Clin Med. 2021;10:9.

17. The Novel Coronavirus Pneumonia Emergency Response Epidemiology Team. The Epidemiological Characteristics of an Outbreak of 2019 Novel Coronavirus Diseases (COVID-19) - China, 2020. China CDC Wkly. 2020;2:113-22.

18. Yang Y, Sugimoto JD, Halloran ME, Basta NE, Chao DL, Matrajt L, et al. The transmissibility and control of pandemic influenza A (H1N1) virus. Science. 2009;326:729-33.

19. Smith DR. Herd Immunity. Vet Clin North Am Food Anim Pract. 2019;35:593-604.

20. Chen Y, Wang AH, Yi B, Ding KQ, Wang HB, Wang JM, et al. Epidemiological characteristics of infection in COVID-19 close contacts in Ningbo city. Zhonghua Liu Xing Bing Xue Za Zhi. 2020;41:667-71.

21. He D, Zhao S, Lin Q, Zhuang Z, Cao P, Wang MH, et al. The relative transmissibility of asymptomatic COVID-19 infections among close contacts. Int J Infect Dis. 2020;94:145-7.

22. Gulland A. Could you be a coronavirus super spreader? http://www. telegraph.co.uk/health-fitness/body/could-coronavirus-super-sprea der/2020. Accessed 16 July 2021.

23. Mizumoto K, Kagaya K, Zarebski A, Chowell G, Estimating the asymptomatic proportion of coronavirus disease,. (COVID-19) cases on board the Diamond Princess cruise ship, Yokohama, Japan, 2020. Euro Surveill. 2019;2020:25.

24. Nishiura H, Kobayashi T, Miyama T, Suzuki A, Jung SM, Hayashi K, et al. Estimation of the asymptomatic ratio of novel coronavirus infections (COVID-19). Int J Infect Dis. 2020;94:154-5.

25. Guan WJ, Ni ZY, Hu Y, Liang WH, Ou CQ, He JX, et al. Clinical Characteristics of Coronavirus Disease 2019 in China. N Engl J Med. 2020;382:1708-20

26. Lauer SA, Grantz KH, Bi Q, Jones FK, Zheng Q, Meredith HR, et al. The Incubation Period of Coronavirus Disease 2019 (COVID-19) From Publicly Reported Confirmed Cases: Estimation and Application. Ann Intern Med. 2020;172:577-82.

27. Linton NM, Kobayashi T, Yang Y, Hayashi K, Akhmetzhanov AR, Jung SM, et al. Incubation period and other epidermiological characteritics of 2019 novel coronavirus infections with right truncation: a statistical analysis of publicly available case data. J Clin Med. 2019;2020:9.

28. Kwok KO, Wong VWY, Wei WI, Wong SYS, Tang JW. Epidemiological characteristics of the first 53 laboratory-confirmed cases of COVID-19 epidemic in Hong Kong, 13 February 2020. Euro Surveill. 2020;25:45.
29. Huang C, Wang Y, Li X, Ren L, Zhao J, Hu Y, et al. Clinical features of patients infected with 2019 novel coronavirus in Wuhan. China Lancet. 2020:395:497-506.

30. Thompson RN. Novel Coronavirus Outbreak in Wuhan, China, 2020: intense surveillance is vital for preventing sustained transmission in New Locations. J Clin Med. 2020;2020:9.

31. Li Q, Guan X, Wu P, Wang X, Zhou L, Tong Y, et al. Early Transmission Dynamics in Wuhan, China, of Novel Coronavirus-Infected Pneumonia. N Engl J Med. 2020;382:1199-207.

32. Hu Z, Song C, Xu C, Jin G, Chen Y, Xu X, et al. Clinical characteristics of 24 asymptomatic infections with COVID-19 screened among close contacts in Nanjing. China Sci China Life Sci. 2020;63:706-11.

33. Lee GJ, Quan FS. Protection induced by early stage vaccination with pandemic influenza virus-like particles. Vaccine. 2016;34:3764-72.

34. Sun Y, Bian C, Xu K, Hu W, Wang T, Cui J, et al. Immune protection induced on day 10 following administration of the 2009 A/H1N1 pandemic influenza vaccine. PLOS ONE. 2010;5:e14270.

35. Editors PLO. Expression of concern: Immune protection induced on day 10 following administration of the 2009 A/H1N1 pandemic influenza vaccine. PLoS One. 2012;7:e39314.

36. Helfand RF, Kebede S, Gary HE Jr, Beyene H, Bellini WJ. Timing of development of measles-specific immunoglobulin $M$ and $G$ after primary measles vaccination. Clin Diagn Lab Immunol. 1999;6:178-80.

37. Helfand RF, Gary HE Jr, Atkinson WL, Nordin JD, Keyserling HL, Bellini WJ. Decline of measles-specific immunoglobulin $\mathrm{M}$ antibodies after primary measles, mumps, and rubella vaccination. Clin Diagn Lab Immunol. 1998;5:135-8.

38. Dawood FS, Chung JR, Kim SS, Zimmerman RK, Nowalk MP, Jackson ML, et al. Interim Estimates of 2019-20 Seasonal Influenza Vaccine Effectiveness - United States, February 2020. MMWR Morb Mortal Wkly Rep. 2020;69:177-82.

39. Rolfes MA, Flannery B, Chung JR, O'Halloran A, Garg S, Belongia EA, et al. Effects of Influenza Vaccination in the United States During the 2017-2018 Influenza Season. Clin Infect Dis. 2019;69:1845-53.

40. Flannery B, Chung JR, Thaker SN, Monto AS, Martin ET, Belongia EA, et al. Interim Estimates of 2016-17 Seasonal Influenza Vaccine Effectiveness - United States, February 2017. MMWR Morb Mortal Wkly Rep. 2017:66:167-71.

41. Buchan SA, Booth S, Scott AN, Simmonds KA, Svenson LW, Drews SJ, et al. Effectiveness of Live Attenuated vs Inactivated Influenza Vaccines in Children During the 2012-2013 Through 2015-2016 Influenza Seasons in Alberta, Canada: A Canadian Immunization Research Network (CIRN) Study. JAMA Pediatr. 2018;172:e181514.

42. Osterholm MT, Kelley NS, Sommer A, Belongia EA. Efficacy and effectiveness of influenza vaccines: a systematic review and meta-analysis. Lancet Infect Dis. 2012;12:36-44.

43. VanderEnde K, Gacic-Dobo M, Diallo MS, Conklin LM, Wallace AS. Globa Routine Vaccination Coverage - 2017. MMWR Morb Mortal Wkly Rep. 2018:67:1261-4.

44. Griffin DE. Measles Vaccine. Viral Immunol. 2018;31:86-95.

45. Rosenthal SR, Clements CJ. Two-dose measles vaccination schedules. Bull World Health Organ. 1993;71:421-8.

46. Doshi RH, Mukadi P, Shidi C, Mulumba A, Hoff NA, Gerber S, et al. Field evaluation of measles vaccine effectiveness among children in the Democratic Republic of Congo. Vaccine. 2015;33:3407-14.

47. Measles vaccines: WHO position paper. Wkly Epidemiol Rec. 2009:84:349-60.

48. Kidd S, Ouedraogo B, Kambire C, Kambou JL, McLean H, Kutty PK, et al. Measles outbreak in Burkina Faso, 2009: a case-control study to determine risk factors and estimate vaccine effectiveness. Vaccine. 2012;30:5000-8.

49. Sudfeld CR, Navar AM, Halsey NA. Effectiveness of measles vaccination and vitamin A treatment. Int J Epidemiol. 2010;39(Suppl 1):i48-55.

50. Singh J, Datta KK. Measles vaccine efficacy in India: a review. J Commun Dis. 1997;29:47-56.

51. Organization WH. The Sinopharm COVID-19 vaccine: What you need to know 2020 https://www.who.int/news-room/feature-stories/detail/thesinopharm-covid-19-vaccine-what-you-need-to-know Accessed 4 Oct 2021. 
52. Zhang J, Litvinova M, Liang Y, Wang Y, Wang W, Zhao S, et al. Changes in contact patterns shape the dynamics of the COVID-19 outbreak in China. Science. 2020;368:1481-6.

53. Chen S, Yang D, Liu R, Zhao J, Yang K, Chen T. Estimating the transmissibility of hand, foot, and mouth disease by a dynamic model. Public Health. 2019;174:42-8.

54. Zhang S, Hu Q, Deng Z, Hu S, Liu F, Yu S, et al. Transmissibility of acute haemorrhagic conjunctivitis in small-scale outbreaks in Hunan Province. China Sci Rep. 2020;10:119.

55. Zhao ZY, Chen Q, Zhao B, Hannah MN, Wang N, Wang YX, et al. Relative transmissibility of shigellosis among male and female individuals: a modeling study in Hubei Province. China Infect Dis Poverty. 2020;9:39.

56. Liu ZH, Zhao YJ, Feng Y, Zhang Q, Zhong BL, Cheung T, et al. Migrant workers in China need emergency psychological interventions during the COVID-19 outbreak. Global Health. 2020;16:75.

57. Chen H, Chen Y, Lian Z, Wen L, Sun B, Wang P, et al. Correlation between the migration scale index and the number of new confirmed coronavirus disease 2019 cases in China. Epidemiol Infect. 2020;148:e99.

58. Wu JT, Leung K, Leung GM. Nowcasting and forecasting the potential domestic and international spread of the 2019-nCoV outbreak originating in Wuhan, China: a modelling study. Lancet. 2020;395:689-97.

59. Hellewell J, Abbott S, Gimma A, Bosse NI, Jarvis Cl, Russell TW, et al. Feasibility of controlling COVID-19 outbreaks by isolation of cases and contacts. Lancet Glob Health. 2020;8:e488-96.

60. Bi Q, Wu Y, Mei S, Ye C, Zou X, Zhang Z, et al. Epidemiology and transmission of COVID-19 in 391 cases and 1286 of their close contacts in Shenzhen, China: a retrospective cohort study. Lancet Infect Dis. 2020;20:911-9.

61. Li C, Ji F, Wang L, Wang L, Hao J, Dai M, et al. Asymptomatic and Humanto-Human Transmission of SARS-CoV-2 in a 2-Family Cluster, Xuzhou. China Emerg Infect Dis. 2020;26:1626-8.

62. Chan JF, Yuan S, Kok KH, To KK, Chu H, Yang J, et al. A familial cluster of pneumonia associated with the 2019 novel coronavirus indicating person-to-person transmission: a study of a family cluster. Lancet. 2020;395:514-23.

63. Nguyen JL, Yang W, Ito K, Matte TD, Shaman J, Kinney PL. Seasonal Influenza Infections and Cardiovascular Disease Mortality. JAMA Cardiol. 2016;1:274-81.

64. Worby CJ, Chaves SS, Wallinga J, Lipsitch M, Finelli L, Goldstein E. On the relative role of different age groups in influenza epidemics. Epidemics. 2015;13:10-6.

65. Lan FY, Wei CF, Hsu YT, Christiani DC, Kales SN. Work-related COVID-19 transmission in six Asian countries/areas: A follow-up study. PLoS ONE. 2020;15:e0233588.

66. Wang D, Hu B, Hu C, Zhu F, Liu X, Zhang J, et al. Clinical Characteristics of 138 Hospitalized Patients With 2019 Novel Coronavirus-Infected Pneumonia in Wuhan. China JAMA. 2020;323:1061-9.

67. Qiu H, Wu J, Hong L, Luo Y, Song Q, Chen D. Clinical and epidemiological features of 36 children with coronavirus disease 2019 (COVID-19) in Zhejiang, China: an observational cohort study. Lancet Infect Dis. 2020;20:689-96.

68. Fan J, Liu X, Pan W, Douglas MW, Bao S. Epidemiology of Coronavirus Disease in Gansu Province, China, 2020. Emerg Infect Dis. 2020;26:1257-65.

69. Jia JW, Ding J, Liu SY, Liao GD, Li JZ, Duan B, et al. Modeling the Control of Covid-19: impact of policy interventions and meteorological factors. Electron J Differ Eq. 2020;9:78.

Ready to submit your research? Choose BMC and benefit from:

- fast, convenient online submission

- thorough peer review by experienced researchers in your field

- rapid publication on acceptance

- support for research data, including large and complex data types

- gold Open Access which fosters wider collaboration and increased citations

- maximum visibility for your research: over $100 \mathrm{M}$ website views per year

At BMC, research is always in progress.

Learn more biomedcentral.com/submissions 